\title{
Mini-Spoilers for Afterbody Base Drag Reduction
}

\author{
D. S. Bulathsinghala ${ }^{1}$, Z. Wang ${ }^{2}$ and I. Gursul ${ }^{3}$ \\ Department of Mechanical Engineering, University of Bath, Bath, BA2 7AY, UK
}

Wind tunnel experiments have been carried out in order to study the effect of full-span minispoilers on the afterbody vortex drag of a slanted base cylinder. Two different protrusion heights $(h / D$ $=2.5 \%$ and $5 \%$ ) were examined at various chordwise locations along the upsweep. The tests were performed on a slanted base cylindrical model with an afterbody upsweep angle $\Phi=28^{\circ}$ at a test Reynolds number of 200,000 based on model diameter. Drag measurements, surface pressure measurements and 2D Particle Image Velocimetry measurements were the main experimental tools utilized within this investigation. Placing the spoilers closer to the leading edge of the upsweep caused an increased drag due to the separation induced by the spoiler itself, leading to a more diffused vortex, but with a larger circulation at the trailing-edge. Drag reductions were observed when placing the spoiler closer to the trailing-edge, with the optimum location being $x_{s}^{\prime} / c=87.5 \%$ resulting in drag reductions of $4.5 \%$ and $4.8 \%$ for $h / D=\mathbf{2 . 5 \%}$ and $5 \%$ heights respectively. This reduction is due to an increase in surface pressure upstream of the spoiler. For the drag reducing spoiler location, the vortex was found to be displaced away from the surface, with the streamlines of the model trailing-edge deflected downwards into the wake of the spoiler, altering the trailing-edge flow. Unsteady aspects are discussed and underlying flow mechanisms are presented using the Proper Orthogonal Decomposition. Further results are also presented for a vortex generator configuration that was examined which may present a useful starting point for future studies. A co-rotating half delta wing type vortex generator placed at an incidence of $\beta=20^{\circ}$ at $x / c=20 \%$ with a leading edge sweep of $\Lambda=7^{\circ}$ was found to show the most promising results.

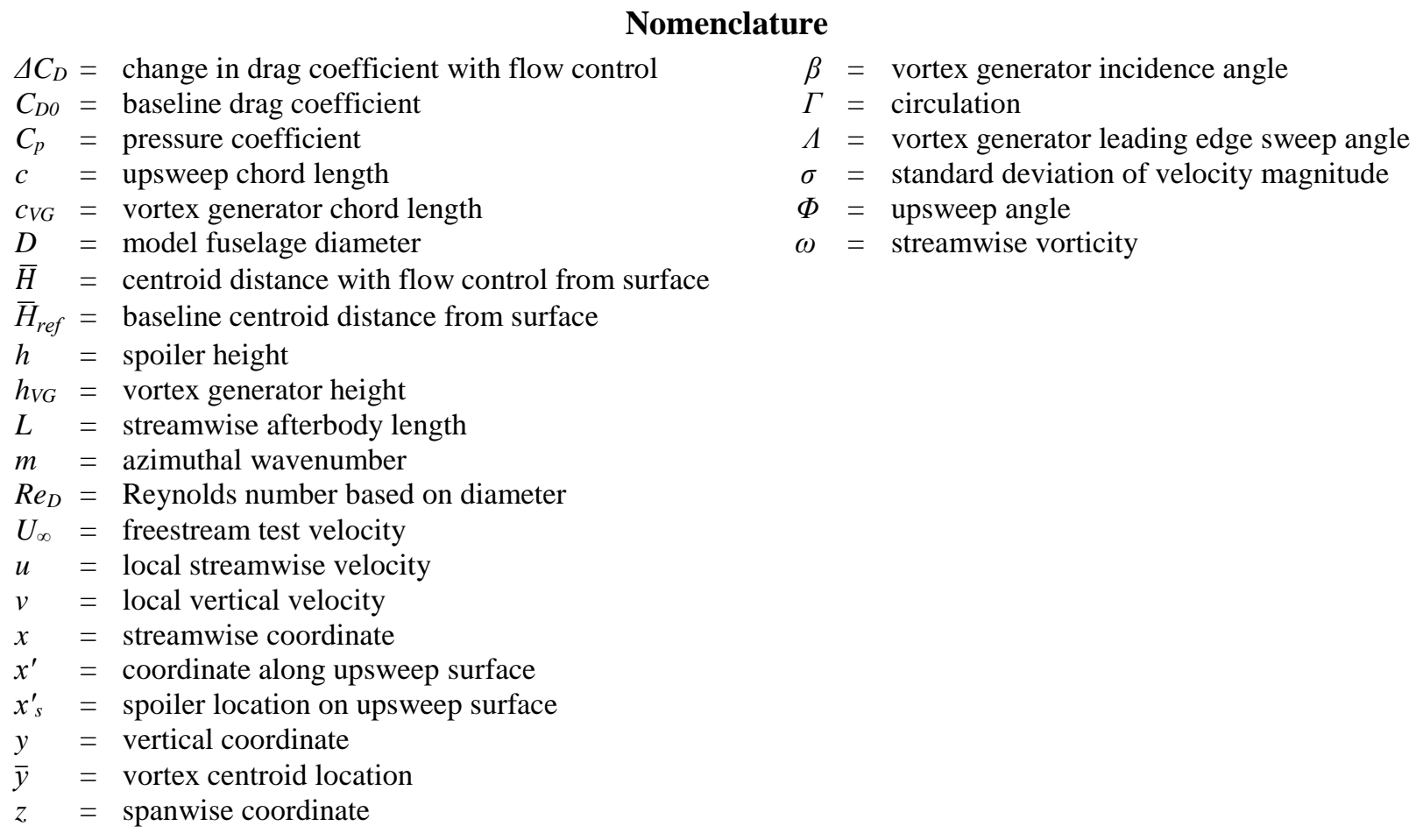

\footnotetext{
${ }^{1}$ Postgraduate Research Student, Department of Mechanical Engineering, Student Member AIAA.

${ }^{2}$ Lecturer, Department of Mechanical Engineering, Member AIAA.

${ }^{3}$ Professor, Department of Mechanical Engineering, Associate Fellow AIAA.
} 


\section{Introduction}

$\mathrm{F}$ USELAGE afterbody vortices are more pronounced on military transport aircraft due to their larger upsweep angles ( $\Phi$, defined in Fig. 1a) and result in an additional pressure drag penalty ${ }^{1}$. In addition to the increased drag, the presence of these vortices result in an upwash towards the centerline of the afterbody, which may interfere with airdrop missions ${ }^{2}$. Due to these reasons, it is desirable to find a solution for this afterbody vortex problem by means of flow control.

The flow physics of afterbody vortex flows were first studied experimentally by means of slanted base cylinder models $\mathrm{s}^{3-6}$. These models enabled a benchmark afterbody vortex flow to be established between different experimental facilities while not being confined to a single aircraft geometry. The results showed that the flowfield and drag coefficient were highly dependent upon the upsweep angle of the afterbody ${ }^{3}$. Two counter-rotating vortices were present at low upsweep angles (below $\Phi=45-50^{\circ}$ depending on experimental arrangement) and a separated wake at higher upsweep angles ${ }^{7}$. A recent experimental campaign initiated at the University of Bath revisited the flow physics of afterbody vortex flows on slanted base cylinders using 2D particle image velocimetry. Tests were performed within two different experimental facilities. Effects of angle of attack and yaw on the baseline flowfield were examined ${ }^{8}$, along with changing the upsweep angle within a range more relevant to military transport aircraft ${ }^{9}$. It was found that the drag coefficient was directly proportional to the trailing-edge vortex strength.

There have been attempts at applying flow control with the aim of drag reduction to afterbody vortex flows. During the development of the Short Belfast aircraft, strakes were placed close to the afterbody trailing edge which resulted in a drag reduction of $7 \%$ as identified from wind tunnel tests ${ }^{10}$. These strakes were thought to disrupt the inflow of air into the afterbody region. Vortex generators are also a common passive flow control approach documented within literature, where a favorable interaction between the generated vortex and the afterbody vortex is sought in order to realize a drag reduction. Calarese et al. ${ }^{11}$ tested a range of passive vortex generators on a scale model of the Lockheed C-130 aircraft, which has an upsweep angle of $28^{\circ}$, and reported drag reductions for specific configurations. Wortmann ${ }^{12}$ tested the application of vortex generators on scale models of Boeing 747 and Lockheed C5 aircraft afterbodies, reporting drag reductions of 3\% and 6\% respectively. Lockheed Martin carried out their own drag reduction study on the $\mathrm{C}-130$ aircraft ${ }^{13}$, investigating the potential of retrofittable microvanes to reduce afterbody drag. The combined computational study and flight test presented beneficial configurations in terms of direct fuel savings to the operator of the aircraft. The primary advantage of these microvanes over conventional vortex generators was their ability to not interfere with airdropping and paratrooping missions. A similar investigation that continued to study the potential of finlets and microvanes was computationally carried out by Telli et al. ${ }^{14}$, suggesting total aircraft drag reductions of $4 \%$ for the best microvane configuration. There have been limited studies applying active flow control to the afterbody vortex problem. Jackson et al. ${ }^{15}$ applied different configurations of blowing jets and slots on a $\Phi=$ $28^{\circ}$ slanted base cylinder model at a Reynolds number of 20,000 (based on model diameter) in a water tunnel. The best results were obtained for a jet flap parallel to the freestream, giving a 9\% drag reduction and 3\% overall energy saving.

The current study is an experimental investigation into the use of spoilers as a passive flow control technique in order to reduce afterbody vortex drag on a slanted base cylinder model with an upsweep angle $\Phi=28^{\circ}$. The results obtained with such a spoiler were presented within the review paper by Bearman ${ }^{7}$, showing the destruction of the vortex footprint for an upsweep angle of $50^{\circ}$ on a slanted base cylinder. Similar spoiler applications have been reported on Ahmed bodies ${ }^{16}$, which is an automotive related bluff body model with a truncated aft end that generates a counterrotating vortex flow structure at slant angles below about $30^{\circ}$. The flow features within the vortex flow regime are similar to the afterbody vortex considered in the current study. Beaudoin and Aider ${ }^{17}$ considered an Ahmed body with a $30^{\circ}$ slant angle, and studied the effect of a spoiler on the junction of the roof and rear slant, reporting $15 \%$ drag reduction for the best deflection angle. Fourrie et al. ${ }^{18}$ tested a similar spoiler at the same location, and reported a drag reduction of $9 \%$ for a base slant angle of $25^{\circ}$. For both studies, the optimum deflector angle was such that the spoiler was almost parallel to the freestream. The spoilers utilized in the current study are perpendicular to the upsweep surface and are of full span at each location. Results are presented in terms of force measurements, pressure measurements and 2D particle image velocimetry measurements. Some preliminary results for a passive vortex generator considered for the current application is also presented at the end of the paper for the interest of the reader and as a potential starting point for future flow control studies.

\section{Experimental Techniques}

The experiments were carried out within the closed return wind tunnel at the Department of Mechanical Engineering, University of Bath. The tunnel has an octagonal cross section with overall dimensions $2.13 \mathrm{~m} \times 1.52 \mathrm{~m}$ x $2.70 \mathrm{~m}$ with a freestream turbulence intensity below $0.4 \%$. The freestream velocity in the working section was 
monitored using a pitot static probe mounted within the tunnel connected to a digital manometer. The experiments were carried out at zero incidence at $U_{\infty}=15 \mathrm{~ms}^{-1}$, which results in a $R e_{D}=200,000$.

\section{A. Wind Tunnel Model}

The slanted base cylindrical model utilized for the experiment had an afterbody upsweep angle $(\Phi)$ of $28^{\circ}$, similar to that of a Lockheed Martin C130 aircraft. The cylindrical fuselage had a diameter $D=200 \mathrm{~mm}$, which was fabricated from PVC. The pipes were cut at the required upsweep angle and the base was covered using $3 \mathrm{~mm}$ thick PVC sheet in order to create the elliptical base of the upsweep. A nose-cone was 3D printed using solid laser sintering which consisted of an ellipsoid of 2:1 major to minor axis ratio. The model parameters and coordinate axes can be identified in Fig. 1a. A custom made streamlined support structure enabled attaching the model onto a force balance, and allowed pressure taps to be fed through. The combined blockage effect of the model and the support system was $2 \%$ of the working section area.

The upsweep section of the model was equipped with 134 surface pressure taps, each of $1.6 \mathrm{~mm}$ diameter, in order to map the surface pressure distribution on the upsweep. The taps were distributed along the starboard side of the upsweep with varying spanwise density according to where the time-averaged vortex was located from initial baseline PIV tests. The locations of the surface pressure taps are shown in Fig. 1b.

A schematic of the spoilers tested in the current study is presented in Fig. 2. It was hypothesized that these spoilers would either disrupt the vortex formation directly or act as flow deflectors which alter the overall flowfield. The spoilers were perpendicular to the surface and were of full span locally at each chordwise location. Two protrusion heights with $h / D=2.5 \%$ and $5 \%$ were tested. The spoilers were tested at 20 chordwise locations along the upsweep, from $x_{s}^{\prime} / c=2.5 \%$ upto $x_{s}^{\prime} / c=97.5 \%$ with a spacing of $\Delta x_{s}^{\prime} / c=5 \%$ in-between locations. The spoilers were 3D printed using ABS material and placed onto the model surface using double sided tape.

\section{B. Force Measurements}

A single component binocular type strain gauge force balance was utilized to measure the total drag force on the model. This type of design consists of two stress concentration throats onto which the strain gauges are mounted, enabling the measurement of the drag force upon calibration. Drag data was acquired at a sampling frequency of 1 $\mathrm{kHz}$ for $10 \mathrm{~s}$ with a minimum of 6 repeats at each flow control configuration. Interesting cases were repeated on varying days in order to assess the uncertainty in measurement and to check its repeatability. The maximum uncertainty in the measurement of $\Delta C_{D}$ was $2 \%$ using the methods outlined by Moffat ${ }^{19}$.

\section{Pressure Measurements}

The time-averaged pressure measurements were performed using a 48 port Scanivalve ${ }^{\circledR}$ multiplexer, connected to a Sensortechnics HCX series differential pressure transducer with an operating range between -10 mbar to +10 mbar. The 134 taps were divided into 3 sets, with the overall surface pressure distribution obtained by combining the three measurements. Each mean pressure measurement consisted of three consecutive repeats, with 1000 readings per tap in each repeat acquired at $1 \mathrm{kHz}$, allowing for a reasonable mean value to be obtained.

\section{Particle Image Velocimetry}

The 2D PIV system utilized for the experiments was a commercial TSI ${ }^{\circledR}$ system which consists of a TSI ${ }^{\circledR} 610036$ synchroniser connected to a $200 \mathrm{~mJ}$ Nd:YAG laser. A six jet TSI ${ }^{\circledR} 9307$ oil droplet generator was used to seed the wind tunnel, where the resulting mean seeding particle diameter was about $1 \mu \mathrm{m}$.

Crossflow PIV measurements were performed on the model at 5 different stations along the afterbody at $x / L=0.2$, 0.4, 0.6, 0.8 and 1.0, which can be identified in Fig. 1a. Image capturing was achieved using a $105 \mathrm{~mm} \mathrm{f} / 2.8 \mathrm{D}$ Nikon lens attached to a 8MP Powerview Plus CCD camera capturing 500 instantaneous image pairs at a capture frequency of about $3.75 \mathrm{~Hz}$. Image processing was achieved using the $\mathrm{TSI}^{\circledR}$ Insight $3 \mathrm{G}$ software, the Hart cross-correlation algorithm was applied with a $48 \times 48$ interrogation area with $50 \%$ overlap. Spatial resolution varied between $0.9 \mathrm{~mm}$ and $1.4 \mathrm{~mm}$ between measurements, less than $1 \%$ of model fuselage diameter. Assuming model alignment and flow symmetry, the starboard side vortex was captured in the crossflow PIV results. The PIV camera was positioned downstream of the model within a transparent perspex box with the laser mounted on a traverse system perpendicular to the freestream (Fig. 3a).

In addition to the crossflow PIV planes, measurements were made on the streamwise $(z=0)$ plane in order to examine the regions of separation induced by spoiler application. The camera was mounted outside the tunnel working section on the starboard side which was equipped with viewing windows. The laser sheet was shined underneath the tunnel working section (Fig. 3b). Within this plane, image capturing was achieved using a $50 \mathrm{~mm}$ f/1.8D Nikon lens. 
PIV image processing settings were the same as before, allowing for $4 \mathrm{~mm}$ spatial resolution. The estimated uncertainty for velocity measurements was $2 \%$ of the freestream velocity.

\section{Results and Discussion}

\section{A. Drag Force}

Figure 4 shows the change in drag coefficient with flow control, normalized against the baseline drag coefficient such that data lying below the horizontal axis represents a drag reduction. Placing the spoiler closer to the leading edge of the upsweep has caused a large increase in the resulting drag coefficient, as much as $19 \%$ increase at $x_{s}^{\prime} / c=$ $2.5 \%$ for the $h / D=5 \%$ spoiler. At around mid-chord, $x_{s}^{\prime} / c=47.5 \%$, the $h / D=2.5 \%$ spoiler shows almost no change in drag coefficient compared to the baseline. On placing the spoiler further downstream, the resulting drag penalty decreases gradually until drag reductions are achieved. The maximum drag reduction for both heights occur at $x_{s}^{\prime} / c=$ $87.5 \%$, with a reduction of $4.5 \%$ for $h / D=2.5 \%$ and $4.8 \%$ for $h / D=5 \%$ compared to the baseline. Beyond this location, the magnitude of the drag reduction decreases towards the trailing-edge for both heights, with a drag reduction of $3.3 \%$ for $h / D=2.5 \%$ and $3.9 \%$ for $h / D=5 \%$ at $x_{s}^{\prime} / c=97.5 \%$. Beyond the optimum location $x_{s}^{\prime} / c=$ $87.5 \%$, the effect of spoiler height $h / D$ on drag reduction is negligible. In light of this drag data, the $x_{s}^{\prime} / c=2.5 \%$, $47.5 \%$ and $87.5 \%$ locations are discussed in detail in the following sections.

\section{B. Pressure Measurements}

Figure 5 presents the surface pressure contours for $x_{s}^{\prime} / c=2.5 \%, 47.5 \%$, and $87.5 \%$ locations along with the baseline for comparison. These plots show a view directly looking at the upsweep surface, and corresponding spoiler locations are denoted by horizontal red lines. The baseline pressure measurement (Fig. 5a) identifies the vortex footprint towards the edge of the elliptical upsweep, which exists until about $x^{\prime} / c=40 \%$. This vortex footprint is the reason for the vortex drag since it represents the lowest pressures acting on the upsweep surface due to the vortex. Further discussions about the same baseline flowfield has been published previously ${ }^{9}$. For the spoiler placed at $x_{s}^{\prime} / c$ $=2.5 \%$ (Fig. 5 b), there exists a large region of low pressure downstream of the spoiler due to flow separation induced by its application. A wider vortex footprint also exists when compared to the baseline. Placing the spoiler closer to the mid-chord of the upsweep, $x_{s}^{\prime} / c=47.5 \%$ (Fig. 5c), appears to have caused a destruction in the vortex footprint to a certain extent. However, downstream of the spoiler, a region of low pressure is present across the span, although not as drastic as with the $x_{s}^{\prime} / c=2.5 \%$ location discussed previously.

Figure $5 \mathrm{~d}$ presents the surface pressure measurements for the optimum location of $x_{s}^{\prime} / c=87.5 \%$. For this drag reducing configuration, an increase in surface pressure is evident just upstream of the spoiler. Figure 6 presents the centerline $(z=0)$ pressure profiles for the same spoiler configurations compared with the baseline measurement. These identify the increase in pressure upstream of each spoiler location along with the low pressure separation region downstream of the spoiler. At the most upstream location $x_{s}^{\prime} / c=2.5 \%$ (Fig. 6a), there exists a large low pressure region downstream of the spoiler, resulting in the large drag penalty. Closer to the mid-chord at $x_{s}^{\prime} / c=47.5 \%$ (Fig. $6 \mathrm{~b})$, the low pressure region downstream of the spoiler is balanced to a certain extent by the increase in surface pressure ahead of the spoiler, resulting in a smaller drag penalty (or almost no drag penalty for the $h / D=2.5 \%$ height). At the optimum location, $x_{s}^{\prime} / c=87.5 \%$ (Fig. 6c), there exists a large positive pressure ahead of the spoiler, which is larger in magnitude than for the previous locations, although this was not immediately apparent on the surface pressure distributions in Fig. 5d. Furthermore, due to the elliptical shape of the upsweep, there is a much smaller surface area downstream of the spoiler for the low pressure separated flow to influence the drag. Flow physics for these configurations will be examined in light of the PIV results in the following sections.

\section{Time-averaged Flowfield}

Figure 7 presents the time-averaged streamwise vorticity for the spoiler placed at $x_{s}^{\prime} / c=2.5 \%$ along with the baseline flowfield for comparison. The results are presented as a 3D perspective view with the flow approaching from right to left, the laser lines intersecting the model surface at each of the measurement locations are denoted by dotted lines.

For the baseline case (Fig. 7a), the vortex formation by means of the shear layer shed from the outboard edge of the afterbody is dominant at the first two measurement stations $x / L=0.2$ and 0.4 . An axisymmetric vortex core region starts appearing beyond the third measurement plane $x / L=0.6$, which gradually starts to move away from the upsweep surface towards the trailing-edge where the fully developed vortex is present. Further details pertinent to this baseline flow can be found in studies published previously ${ }^{8,9}$.

For the $x_{s}^{\prime} / c=2.5 \%$ location (Figs $7 \mathrm{~b}$ and $7 \mathrm{c}$ ), at the most upstream station $(x / L=0.2)$ the vortex formation occurs further outboard in comparison to the baseline due to the presence of flow separation immediately downstream of the 
spoiler. As the vortex approaches the trailing-edge it becomes increasingly diffuse for both spoiler heights, with the vortex appearing more diffuse for the larger spoiler height $h / D=5 \%$ (Fig. 7c). This apparent diffusion could be due to the ingestion of turbulence generated by the spoiler, resulting in an increase in the vortex meandering. Figure 8 presents the flow in the streamwise $(z=0)$ plane for the same spoiler location with flow approaching from left to right, and shows a large extent of flow separation due to the spoiler, contributing to its high drag penalty. The streamlines suggest that the flow reattaches at around mid-chord for both spoiler heights, which is consistent with the pressure distribution observed in Fig. 6a.

Figure 9 presents a 3D perspective view of the time-averaged streamwise vorticity for the spoiler placed at $x_{s}^{\prime} / c=$ $47.5 \%$. The first two measurement planes $x / L=0.2$ and 0.4 for both spoiler heights show very similar flowfields compared to the baseline (Fig. 9a) since the spoiler is located downstream of both these measurement planes. The first measurement plane downstream of the spoiler, $x / L=0.6$, shows that the initial afterbody vortex is destroyed compared to the baseline flow. Remnants of the original vortex can still be seen, along with signs of a new afterbody vortex starting to form close to the surface. In-between these two vortices is a region of opposite (positive) vorticity originating from the spoiler, more prominently observable in Fig. $9 \mathrm{c}$ for $h / D=5 \%$. This interaction results in the formation of a very diffuse vortex structure towards the trailing-edge in comparison to the baseline. The streamwise $(z=0)$ plane in Fig. 10 shows regions of flow separation for both spoiler heights, much less prominent than for the spoiler placed at $x_{s}^{\prime} / c=2.5 \%$ seen in Fig. 8. Hence at this location, a smaller parasitic drag is expected due to the spoiler. For the $h / D=2.5 \%$ case, the parasitic drag has been compensated by the build up of high pressure ahead of the spoiler, leading to a drag coefficient almost equal to the baseline.

The 3D perspective view of time-averaged streamwise vorticity for the optimum location $x_{s}^{\prime} / c=87.5 \%$ is shown in Fig. 11. At first glance there appears to be no appreciable change in vortex structure when the spoiler is placed at this location for both protrusion heights, with very similar time-averaged flowfields in comparison to the baseline. However, the vortex at the trailing-edge appears to have been displaced slightly away from the surface due to the presence of the spoiler, more observable in Fig. 11c with the larger height $(h / D=5 \%)$ spoiler. This effect will be discussed and quantified in the forthcoming discussion. The flow in the streamwise $(z=0)$ plane is presented in Fig. 12 , where a region of flow separation exists downstream of the spoiler. At this location, this low pressure region is close enough to the trailing-edge in order to deflect the streamlines downward into the wake of the spoiler. This feature was not seen with the spoiler locations presented previously in Fig. 8 and Fig. 10, where the trailing-edge streamlines leave the model smoothly. The time-averaged vorticity shows two regions of opposite vorticity which exist close to the trailing-edge of the model.

Having discussed the vortex characteristics using the time-averaged flowfields, it was desirable to quantify the strength of the vortex within each crossflow measurement plane. In order to asses the vortex strength, circulation of each crossflow measurement plane was calculated using a numerical method in MATLAB ${ }^{\circledR}$. Center of the vortex was located initially using the Q-criterion ${ }^{20,21}$. To calculate the Q-criterion, the velocity gradient tensor, $\nabla u$, is first decomposed into symmetrical and asymmetrical components; the strain tensor, $S=0.5\left(\nabla u+\nabla u^{\mathrm{T}}\right)$ and the vorticity tensor, $\Omega=0.5\left(\nabla u-\nabla u^{\mathrm{T}}\right)$. A vortex is present in the region where $Q=0.5\left(\|\Omega\|^{2}-\|S\|^{2}\right)>0$, and the center of the vortex is identified as the location of maximum Q value. The circulation within the immediate neighbourhood of this center was then calculated using an area integral of vorticity, before expanding the area outward along the grid by one spatial resolution unit and recalculating the circulation until the resulting change in circulation was less than $0.5 \%$. The Qcriterion was considered a more robust method of locating the vortex center as opposed to using maximum vorticity since it is capable of separating regions of rotation from regions of strain which may have high vorticity if a shear layer is present. Determining the circulation using the current method overcomes the ambiguity of choosing an arbitrary domain of integration to perform the calculation, furthermore, the influence of background noise is minimal due to the enforced $0.5 \%$ change criterion.

The calculated circulation for the three spoiler locations are shown in Fig. 13. The baseline circulation increases rapidly between $x / L=0.2$ and 0.4 stations due to the strong shear layer present at these measurement planes aiding the vortex formation, and keeps increasing towards the trailing-edge as vorticity is continuously shed into the vortex core by means of the shear layer. The shear layer is still present at the further downstream stations but is weak in comparison to the vortex and hence is not clearly visible in the time-averaged vorticity presented previously. With spoiler application at $x_{s}^{\prime} / c=2.5 \%$ (Fig. 13a), the circulation growth is more rapid. At $x / L=0.6$, both spoiler heights have a larger circulation compared to the baseline and this value stays almost constant until $x / L=1.0$, with the $h / D=$ $2.5 \%$ and $5 \%$ heights resulting in $6 \%$ and $13 \%$ larger circulations compared to the baseline respectively. It is worthy to note that the rapid increase in vortex circulation (until $x / L=0.6$ ) occurs while the flow in the $z=0$ plane is separated (see Fig. 8). With the spoiler closer to the mid-chord at $x_{s}^{\prime} / c=47.5 \%$ (Fig. 13b), the resulting growth in circulation is smaller in magnitude compared to the baseline at the most upstream measurement planes ahead of the spoiler $(x / L=$ 0.2 and 0.4 ), with both spoiler heights showing equal values of circulation. Between $x / L=0.4$ and 0.6 measurement 
planes, there is a clear deceleration in the circulation growth due to the disruption of the shear layer by the spoiler at $x_{s}^{\prime} / c=47.5 \%$. Although there is some recovery at $x / L=0.8$ due to the merging of the two vortices (see Fig. 9), the final circulation at the trailing-edge does not recover back to baseline values. Hence placing the spoiler closer to the mid-chord of the upsweep has resulted in a slightly weaker afterbody vortex at the trailing-edge, with the $h / D=2.5 \%$ and 5\% heights resulting in $4 \%$ and $8 \%$ lower circulations compared to the baseline respectively. A weaker vortex at the trailing-edge is desirable, since it has been shown previously that the vortex strength at the trailing-edge can have direct implications on the overall drag coefficient ${ }^{9}$. However, with the spoiler at this location, this decrease in vortex strength was inadequate to result in a drag reduction due to the parasitic drag of the spoilers discussed previously.

The circulation for the optimum spoiler location $x_{s}^{\prime} / c=87.5 \%$ is shown in Fig. 13c, and can be observed to be almost equal to the baseline circulation at most of the measurement stations for both spoiler heights. This was expected since the time-averaged flowfield encountered in Fig. 11 was very similar to the baseline. It was mentioned during the discussion for Fig. 11 that the vortex seemed to be displaced downwards away from the upsweep trailing-edge with the spoiler application. In order to quantify this, the vortex centroid locations within the crossflow plane were obtained in the y-direction according to Equation $1^{22}$.

$$
\bar{y}=\frac{1}{\Gamma} \iint y \omega d y d z
$$

The first measurement station $x / L=0.2$ was excluded from this analysis as the flow was very much shear layer dominated. Once the centroids were obtained, the distance of each centroid location from the surface of the model was calculated for the baseline $\left(\bar{H}_{r e f}\right)$ and flow control cases $(\bar{H})$ at each crossflow measurement plane. The difference of these two parameters $\left(\bar{H}-\bar{H}_{\text {ref }}\right)$ represent a displacement of the vortex due to flow control, with positive denoting the vortex displacing away from the surface at that particular measurement plane. The results for the spoiler at $x_{s}^{\prime} / c=$ $87.5 \%$ are presented in Fig. 14. At $x / L=0.4$ and 0.6 the vortex is not displaced appreciably compared to the baseline centroid location, however, at $x / L=0.8$ the vortex starts to be displaced downwards away from the surface. At the trailing-edge $x / L=1.0$, the vortex is clearly displaced away from the surface relative to the baseline vortex centroid, with the larger height $h / D=5 \%$ spoiler displacing the vortex further away from the surface compared to the $h / D=$ $2.5 \%$ spoiler. Such a movement of the vortex away from the surface is desirable, since its influence on the surface pressure is reduced. Hence it is expected that this effect has an underlying influence on the drag reduction.

\section{Unsteady Aspects}

Figure 15 presents the 3D views of standard deviation of velocity within the crossflow planes for the spoiler locations discussed previously, along with the baseline case for comparison. This effectively represents the turbulence intensity within the flowfield, and is useful in visualizing unsteady flow features. Only the larger height $h / D=5 \%$ cases are presented since the $h / D=2.5 \%$ cases showed similar flowfields. For the baseline in Fig. 15a, high levels of standard deviation are observed in regions where vortex meandering occurs. The shear layer that was not visible downstream of $x / L=0.6$ in the time-averaged flow is now visible due to the flow unsteadiness. With the spoiler placed at $x_{s}^{\prime} / c=2.5 \%$ (Fig. 15b), the highly unsteady flow generated by the spoiler is clearly visible at the first three measurement planes from $x / L=0.2$ to $x / L=0.6$. Towards $x / L=0.8$ and 1.0 , the magnitude of the unsteadiness decreases since the flow is now reattached to the surface. The resulting vortex has ingested the turbulence generated from the spoiler, resulting in a large region of unsteadiness at the final two measurement stations which caused an apparent diffusion in the time-averaged vorticity.

For the spoiler placed at $x_{s}^{\prime} / c=47.5 \%$ (Fig. 15c) the first measurement plane $x / L=0.2$ looks very similar to the baseline since the effect of the spoiler is not felt at this location. At $x / L=0.4$, there appears to have been a reduction in the peak unsteadiness closer to the vortex core location, with an increased unsteadiness towards the upsweep centerline as the flow approaches the spoiler location. Downstream of the spoiler at $x / L=0.6$, the turbulent wake of the spoiler is visible. Beyond this location, similar to the spoiler at $x_{s}^{\prime} / c=2.5 \%$, the vortex ingests this turbulence as it propagates to $x / L=0.8$ and 1.0 , resulting in an increase in its meandering and appearing highly diffuse in the timeaveraged sense (Fig. 9). For the $x_{s}^{\prime} / c=87.5 \%$ spoiler location (Fig. 15d), the unsteadiness appears very similar to the baseline for all measurement planes upto $x / L=0.8$. The final measurement plane at the trailing edge, $x / L=1.0$, shows the turbulence generated by the spoiler at this location, the vortex displacement away from the surface is also apparent with the vortex core unsteadiness located further away from the surface in comparison to the baseline. 


\section{E. Proper Orthogonal Decomposition}

The Proper Orthogonal Decomposition (POD) is a mathematical means of extracting the underlying flow features (or modes) within a flowfield ${ }^{23}$. The current analysis was performed using a MATLAB ${ }^{\circledR}$ code that employs the method of snapshots ${ }^{24,25}$. The POD modes of the baseline case have been extensively discussed in previous studies ${ }^{9}$ which revealed the dominant $m=1$ helical displacement mode at the trailing-edge for the fully developed afterbody vortex. This same $m=1$ mode had previously been identified with wing tip vortices ${ }^{26,27}$, delta wing vortices ${ }^{28}$ and inlet vortices ${ }^{29}$.

The POD results for the $h / D=5 \%$ cases discussed previously are presented in Fig. 16, only the most dominant POD mode is presented in each measurement plane. The $m=1$ helical mode can be identified by the presence of the vortex dipole for the baseline case at the trailing-edge $x / L=1.0$ in Fig. 16a, corresponding to $14 \%$ of the total energy. This vortex dipole is centered around the corresponding location of the time-averaged vorticity, the effect of which is to increase vorticity on one half of the time-averaged vortex and decrease it on the other, resulting in a displacement of the core ${ }^{30}$. Hence the $m=1$ mode can be thought to represent the dominant mechanism of meandering within the crossflow plane. For the spoiler at $x_{s}^{\prime} / c=2.5 \%$ in Fig. 16b, at the first two measurement planes $x / L=0.2$ and 0.4 , the dominant POD mode is very different to the baseline since the flow is now dominated by the turbulence due to the spoiler. Beyond $x / L=0.6$, the $m=1$ mode does develop, with the vortex dipole visible at $x / L=1.0$. It contains a higher mode energy percentage compared to the baseline, with $23 \%$ of the total energy. For the $x_{s}^{\prime} / c=47.5 \%$ spoiler in Fig. $16 \mathrm{c}$, the spoiler has disrupted the development of the $m=1$ mode at $x / L=0.6$. However, the $m=1$ mode is present at the trailing-edge and the mode energy for this mode was found to be similar to the baseline energy at the same measurement location. The dominant POD modes for the spoiler at $x_{s}^{\prime} / c=87.5 \%$ appear very similar in terms of energy as the baseline, suggesting that the underlying flow physics are very similar. At the trailing-edge, the vortex dipole representing the $m=1$ mode has been displaced away from the surface owing to the displacement of the vortex discussed previously.

\section{F. Passive Vortex Generator Configurations}

In the interest of applying a different method of flow control to the afterbody vortex problem, the potential of passive vortex generators (VGs) was briefly investigated. The results reported here present the most interesting configuration with potential future benefit, and may provide a starting point for future flow control studies related to afterbodies. The VGs studied were of half delta wing (or vane) type, which were considered over flat plate designs due to the expected possibility of higher VG incidence angles without compromising flow quality. The chord length was fixed at $c_{V G} / D=27.5 \%$, and the half delta wing VG tested had a leading edge sweep $\Lambda=70^{\circ}$, resulting in a VG height to model diameter ratio of $h_{V G} / D=10 \%$. The locations of the VG pairs were varied along the side edges of the circular fuselage on the afterbody on both sides of the model, such that the VG trailing edges were placed (when viewed from a sideview) at $x / c=5 \%, 10 \%$, (and with spacings of $x / c=10 \%$ from there on) up to $x / c=50 \%$ along the afterbody. At each location, the effect of changing the VG incidence angle was investigated for $\beta=20^{\circ}, 30^{\circ}$ and $40^{\circ}$, relative to the freestream direction. VG configurations are usually characterized based on whether a pair are co-rotating or counter-rotating relative to each other ${ }^{31,32}$. However, in the current study, the VGs are characterized based on the rotational sense of the generated vortex in comparison to the afterbody vortex. Force data revealed that the best location with drag reduction potential was $x / c=20 \%$ with a co-rotating VG pair with $\beta=20^{\circ}$. At this configuration, the drag coefficient was equal to the baseline. This suggested that there was an underlying flow mechanism that overcomes the parasitic drag of the VG, the same was not true for the counter-rotating case, which resulted in a drag increase. PIV measurements were performed for this VG location $(x / c=20 \%)$ and incidence angle $\left(\beta=20^{\circ}\right)$ for both co-rotating (beneficial) and counter-rotating (detrimental) cases in order to understand the flow interactions.

Figure 17 presents the 3D perspective views of time-averaged vorticity for the VG cases. For the co-rotating configuration in Fig. 17b, the co-rotating vortex from the VG is captured at the $x / L=0.4$ measurement plane, and is seen to interact with the afterbody vortex at this location, distorting its time-averaged vorticity compared to the baseline. Further downstream, these two vortices appear to merge together and form a diffused vortex at the trailingedge, which appears to be further away from the surface compared to the baseline time-averaged vortex. The counterrotating VG configuration is presented in Fig. 17c, where the counter-rotating vortex generated by the VG is visible at $x / L=0.4$ and 0.6 locations. However, the afterbody vortex flow has not changed significantly in comparison to the baseline. The time-averaged vortex that exists at the trailing-edge, $x / L=1.0$, appears very similar to that of the baseline case. The circulation was calculated for all measurement planes using the same method discussed previously (not shown here). It was found that the growth in circulation was more rapid for the counter-rotating VG, and slower for the co-rotating VG compared to the baseline. However, the circulation for the fully developed vortex at the trailingedge recovered back to baseline values for both cases. The vortex centroid locations were calculated using the same methods described previously for measurement planes downstream of $x / L=0.4$ for both VG configurations, and is 
presented in Fig. 18. It can be observed that the vortex centroid has been displaced away from the surface in comparison to the baseline for the co-rotating case at all measurement locations. This movement of the vortex away from the surface is the reason for a resulting drag coefficient equal to the baseline, with the parasitic drag of the VG being compensated by the movement of the vortex away from the surface, especially at upstream locations where the vortex influence on the surface pressure is the largest (e.g. $x / L=0.4)$. In contrast, the counter-rotating case reveals that the centroids have moved closer to the surface, resulting in a larger suction at the surface leading to a larger drag penalty.

\section{Conclusions}

The efficacy of full span mini-spoilers for afterbody base drag reduction has been studied experimentally in a wind tunnel at a Reynolds number $R e_{D}=200,000$ on a slanted base cylindrical model with a $\Phi=28^{\circ}$ upsweep angle. It was found that placing the spoilers closer to the leading edge of the upsweep created a drag increase due to flow separation downstream of the spoiler, leading to the formation of a stronger but diffused vortex at the trailing-edge. The diffusion of the time-averaged vortex was due to the ingestion of turbulence emanating from the spoiler. Placing the spoiler closer to the mid-chord at $x_{s}^{\prime} / c=47.5 \%$ resulted in a weaker vortex at the trailing-edge, however, drag reductions were not achieved at this location due to the parasitic drag of the spoiler. Moving the spoiler location further downstream, drag reductions are achieved. At the optimum location for drag reduction, $x_{s}^{\prime} / c=87.5 \%$, the $h / D=2.5 \%$ and 5\% heights resulted in drag reductions of $4.5 \%$ and $4.8 \%$ respectively, and this was attributed to the increase in surface pressure ahead of the spoiler. The resulting time-averaged vortex was moved further away from the surface at the trailing-edge compared to the baseline case, along with the trailing-edge streamlines being deflected downwards into the wake of the spoiler. This movement of the vortex away from the surface caused the drag reduction observed for this spoiler location. Proper orthogonal decomposition revealed the dominant helical displacement mode with azimuthal wavenumber $m=1$ at the trailing-edge for all cases. Some preliminary results were also presented for a half delta wing vortex generator with a leading edge sweep $\Lambda=70^{\circ}$ placed at $x / c=20 \%$ with $\beta=20^{\circ}$ incidence. The more potentially beneficial co-rotating configuration displaced the resulting vortex away from the surface, while the counter-rotating configuration forced the vortex closer towards the surface in comparison to the baseline. This could provide a good starting point for future vortex generator studies applied to afterbody vortex flows.

\section{Acknowledgments}

The work presented within this study is sponsored by the United States Airforce Office for Scientific Research (AFOSR) under grant number FA9550-14-1-0126. 


\section{References}

${ }^{1}$ Epstein, R. J., Carbonaro, M. C., and Caudron, F., "Experimental investigation of the flowfield about an upswept afterbody", Journal of Aircraft, Vol. 31, No. 6, 1994, pp. 1281-1290.

${ }^{2}$ Bury, Y., Jardin, T., and Klockner, A., "Experimental investigation of the vortical activity in the close wake of a simplified military transport aircraft", Experiments in Fluids, Vol. 54, No. 5, 2013.

${ }^{3}$ Morel, T., "Effect of base slant on flow in the near wake of an axisymmetric cylinder", Aeronautical Quarterly, Vol. 31, No. 2, 1980, pp. 132-147.

${ }^{4}$ Maull, D. J., "The drag of slant-based bodies of revolution", The Aeronautical Journal, Vol. 84, 1980, pp. 164-166.

${ }^{5}$ Xia, X. J., and Bearman, P. W., "Experimental investigation of the wake of an axisymmetric body with a slanted base", Aeronautical Quarterly, Vol. 34, No. 1, 1983, pp. 24-45.

${ }^{6}$ Britcher, C. P., and Alcorn, C. W., "Interference-free measurements of the subsonic aerodynamics of slanted-base ogive cylinders", AIAA Journal, Vol. 29, No. 4, 1991, pp. 520-525.

${ }^{7}$ Bearman, P. W., "Review - Bluff Body Flows Applicable to Vehicle Aerodynamics", Journal of Fluids Engineering, Transactions of the ASME, Vol. 102, No. 3, 1980, pp. 265-274.

${ }^{8}$ Jackson, R., Wang, Z., and Gursul, I., "Control of Afterbody Vortices by Blowing”, AIAA-2015-2777, 45" AIAA Fluid Dynamics Conference, 22-26 June 2015, Dallas, TX, USA.

${ }^{9}$ Bulathsinghala, D. S., Wang, Z., and Gursul, I., "Effect of Upsweep Angle on Afterbody Vortices", AIAA-2016-4344, 46 AIAA Fluid Dynamics Conference, 13-17 June 2016, Washington, DC, USA.

${ }^{10}$ McCluney, B., and Marshall, J., "Drag development of Belfast”, Aircraft Engineering, Vol. 39, No. 10, 1967, pp. 33-37.

${ }^{11}$ Calarese, W., Crisler, W. P. \& Gustafson, G. L., “Afterbody drag reduction by vortex generators", AIAA-1985-0354, 23 ${ }^{\text {rd }}$ AIAA Aerospace Sciences Meeting, 1985, Reno, NV, USA.

${ }^{12}$ Wortmann, A., "Reduction of fuselage form drag by vortex flows", Journal of Aircraft, Vol 36, No. 3, 1999, pp. $501-506$.

${ }^{13}$ Smith, B. R., Yagle, P. J. \& Hooker, J. R., "Reduction of Aft Fuselage Drag on the C-130 Using Microvanes", AIAA-20130105, $51^{\text {st }}$ AIAA Aerospace Sciences Meeting, 7-10 January 2013, Grapevine, TX, USA.

${ }^{14}$ Telli, H., Ayan, E., Soyer, S., Gülsever, E., and Özcan, S., "An investigation of C-130 aircraft base drag reduction with aftbody modifications," AIAA-2016-3430, 34 th AIAA Applied Aerodynamics Conference, 13-17 June 2016, Washington, DC, USA.

${ }^{15}$ Jackson, R., Wang, Z., and Gursul, I., “Afterbody Drag Reduction Using Active Flow Control”, AIAA-2017-0954, 55 $5^{\text {th }}$ AIAA Aerospace Sciences Meeting, January 2017, Grapevine, TX, USA.

${ }^{16}$ Ahmed, S. R., Ramm, G., and Fatlin, G., "Some salient features of the time-averaged ground vehicle wake", SAE technical paper series, 1984, No. 840300 .

${ }^{17}$ Beaudoin, J. F., and Aider, J. L., "Drag and lift reduction of a 3D bluff body using flaps", Experiments in Fluids, Vol. 44, No. 4, 2008, pp. 491-501.

${ }^{18}$ Fourrie, G., Keirsbulck, L., Labraga, L., and Gillieron, P., "Bluff-body drag reduction using a deflector", Experiments in Fluids, Vol. 50, No. 2, 2011, pp. 385-395.

${ }^{19}$ Moffat, R. J., "Describing the Uncertainties in Experimental Results", Experimental Thermal and Fluid Science, Vol. 1, No. 1, 1988, pp. 3-17.

${ }^{20}$ Hunt, J. C. R., Wray, A. A., and Moin, P., "Eddies, stream and convergence zones in turbulent flows", Center for Turbulence Research Report CTR-S88, Vol. 193, 1988.

${ }^{21}$ Jeong, J., and Hussain, F., "On the identification of a vortex”, Journal of Fluid Mechanics, Vol. 285, 1995, pp. 69-94.

${ }^{22}$ Leweke, T., Le Dizés, S., and Williamson, C. H. K., "Dynamics and instabilities of vortex pairs", Annual Reviews of Fluid Mechanics, Vol. 48, 2016, pp. 507-541.

${ }^{23}$ Berkooz, G., Holmes, P., and Lumley, J. L., "The proper orthogonal decomposition in the analysis of turbulent flows", Annual Reviews of Fluid Mechanics, Vol 25, 1993, pp. 539-575.

${ }^{24}$ Chen, H., Reuss, D. L., and Sick, V., "On the use and interpretation of proper orthogonal decomposition of in-cylinder engine flows", Measurements Science and Technology, Vol 23:085302, 2012.

${ }^{25}$ Chen, H., Hung, D. L. S., Reuss, D. L., and Sick, V., “A practical guide for using proper orthogonal decomposition in engine research”, International Journal of Engine Research, Vol 14, 2013, pp. 307-319.

${ }^{26}$ Del Pino, C., Lopez-Alonso, J. M., Parras, L., Fernandez-Feria, R., "Dynamics of the wing-tip vortex in the near field of a NACA 0012 aerofoil”, The Aeronautical Journal, Vol. 115, No. 1166, 2011, pp. 229-239.

${ }^{27}$ Chen, C., Wang, Z., Cleaver, D. J., and Gursul, I., "Interaction of trailing vortices with downstream wings", AIAA-20161848, 54 ${ }^{\text {th }}$ AIAA Aerospace Sciences Meeting, 2016, San Diego, CA, USA.

${ }^{28}$ Zhang, X., Wang, Z. and Gursul, I., "Interaction of Multiple Vortices over a Double Delta Wing", Aerospace Science and Technology, Vol. 48, 2016, pp. 291-307.

${ }^{29}$ Wang, Z., and Gursul, I., "Unsteady characteristics of inlet vortices", Experiments in Fluids, Vol. 53, 2012, pp. 1015-1032.

${ }^{30}$ Fabre, D., Sipp, D., and Jacquin, L., "Kelvin waves and the singular modes of the Lamb-Oseen vortex", Journal of Fluid Mechanics, Vol. 551, 2006, pp. 235-274.

${ }^{31}$ Ashill, P. R., Fulker, J. L., and Hackett, K. C., "A review of recent developments in flow control”, The Aeronautical Journal, Vol. 109, No. 1095, 2005, pp. 205-232.

${ }^{32}$ Lin, J. C., "Review of research on low-profile vortex generators to control boundary-layer separation", Progress in Aerospace Sciences, Vol. 38, No. 4-5, 2002, pp. 389-420. 


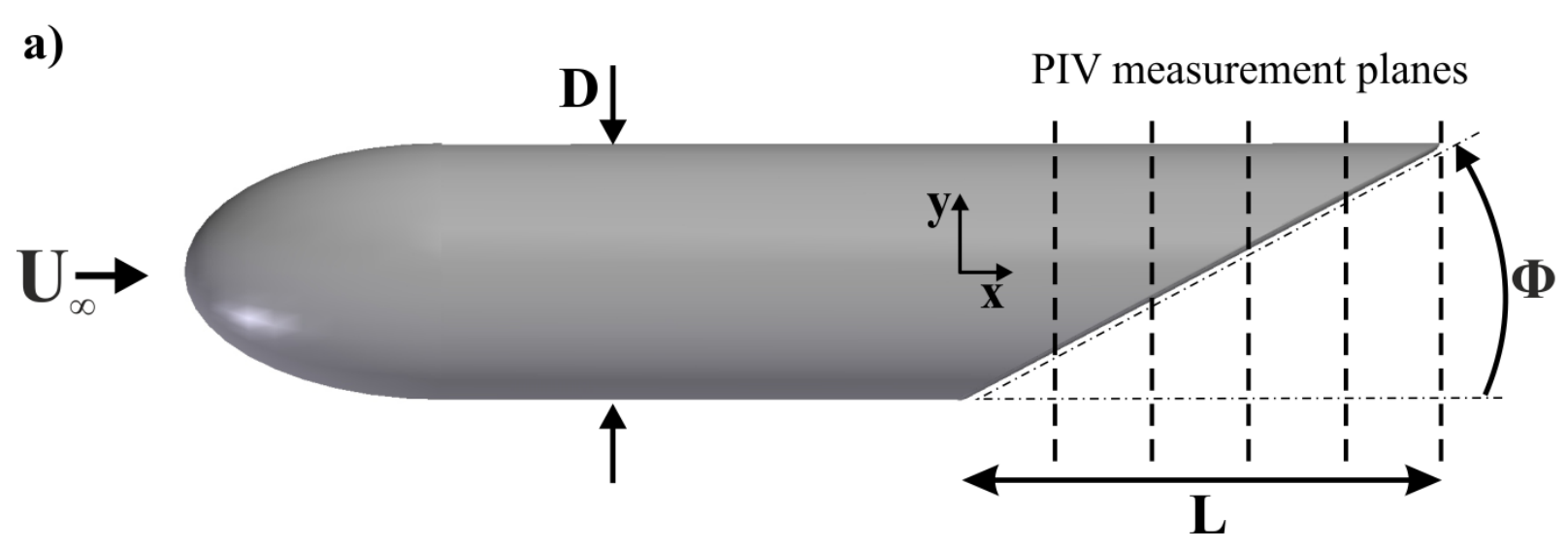

b)

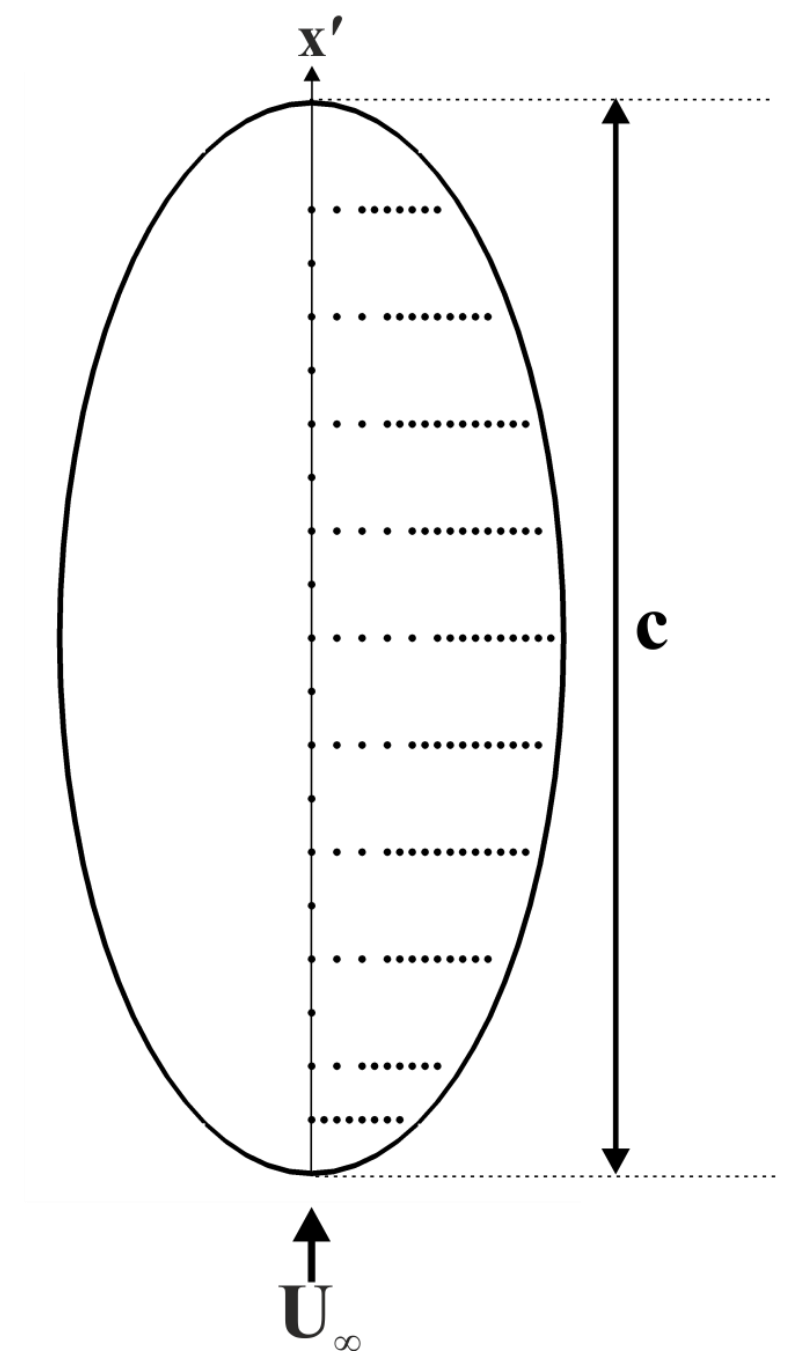

Figure 1. a) Model parameters and crossflow measurement planes, b) Pressure tap locations on upsweep. 
a)

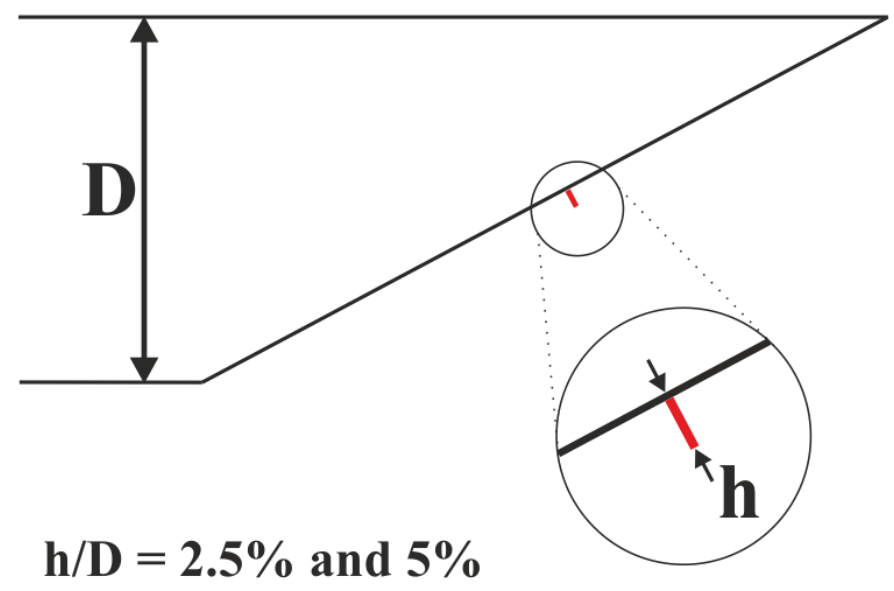

b)

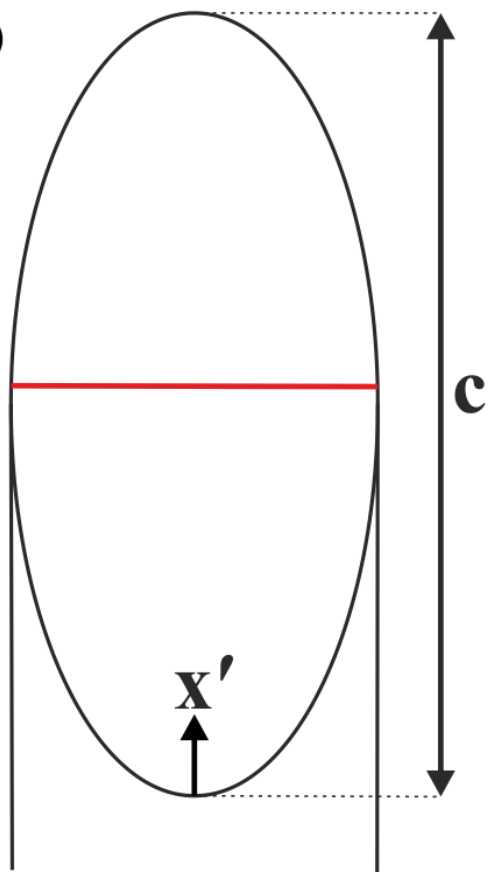

Figure 2. Schematic of full span spoilers placed on the model. a) View from port side, b) View looking at upsweep surface. 


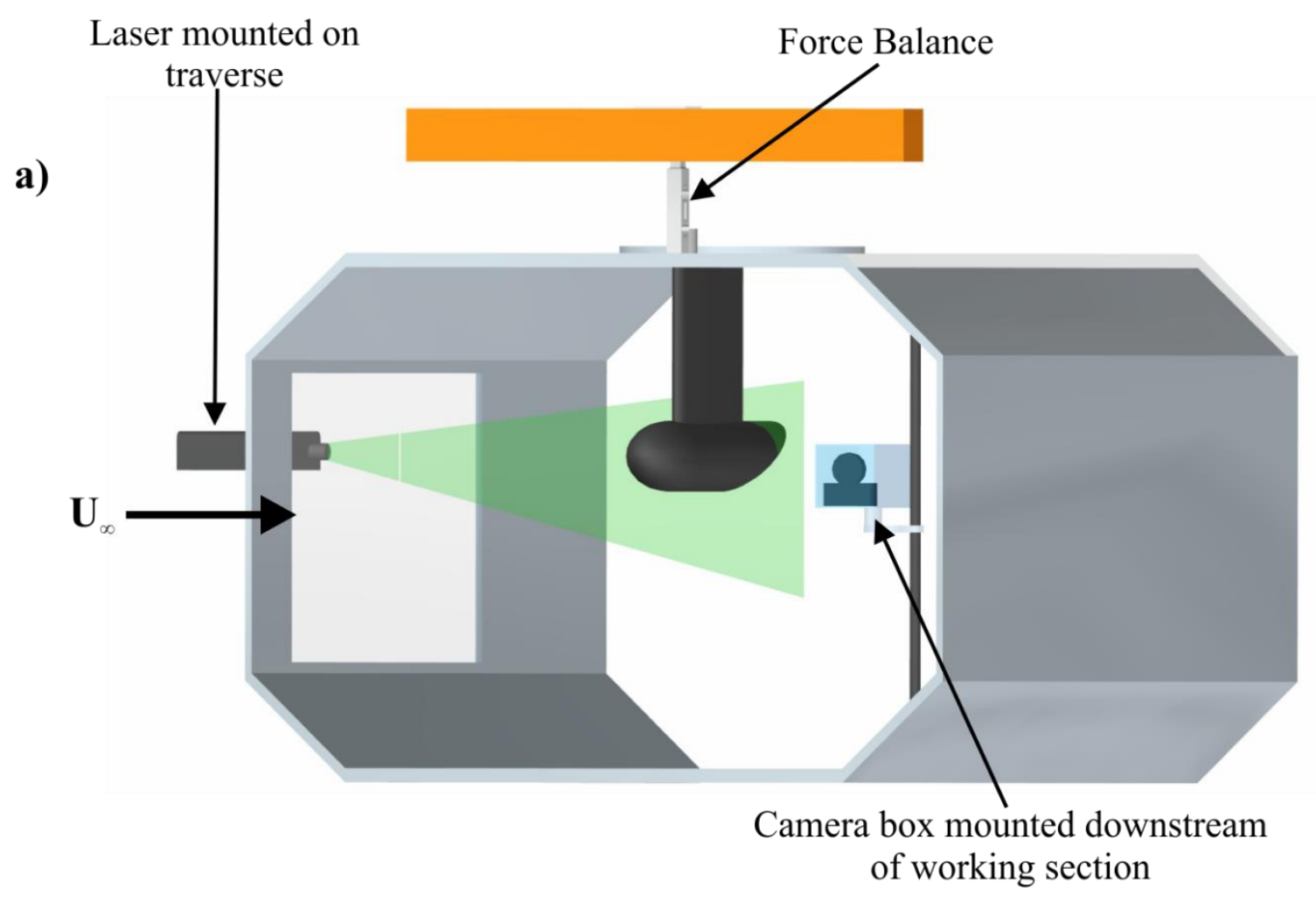

b)

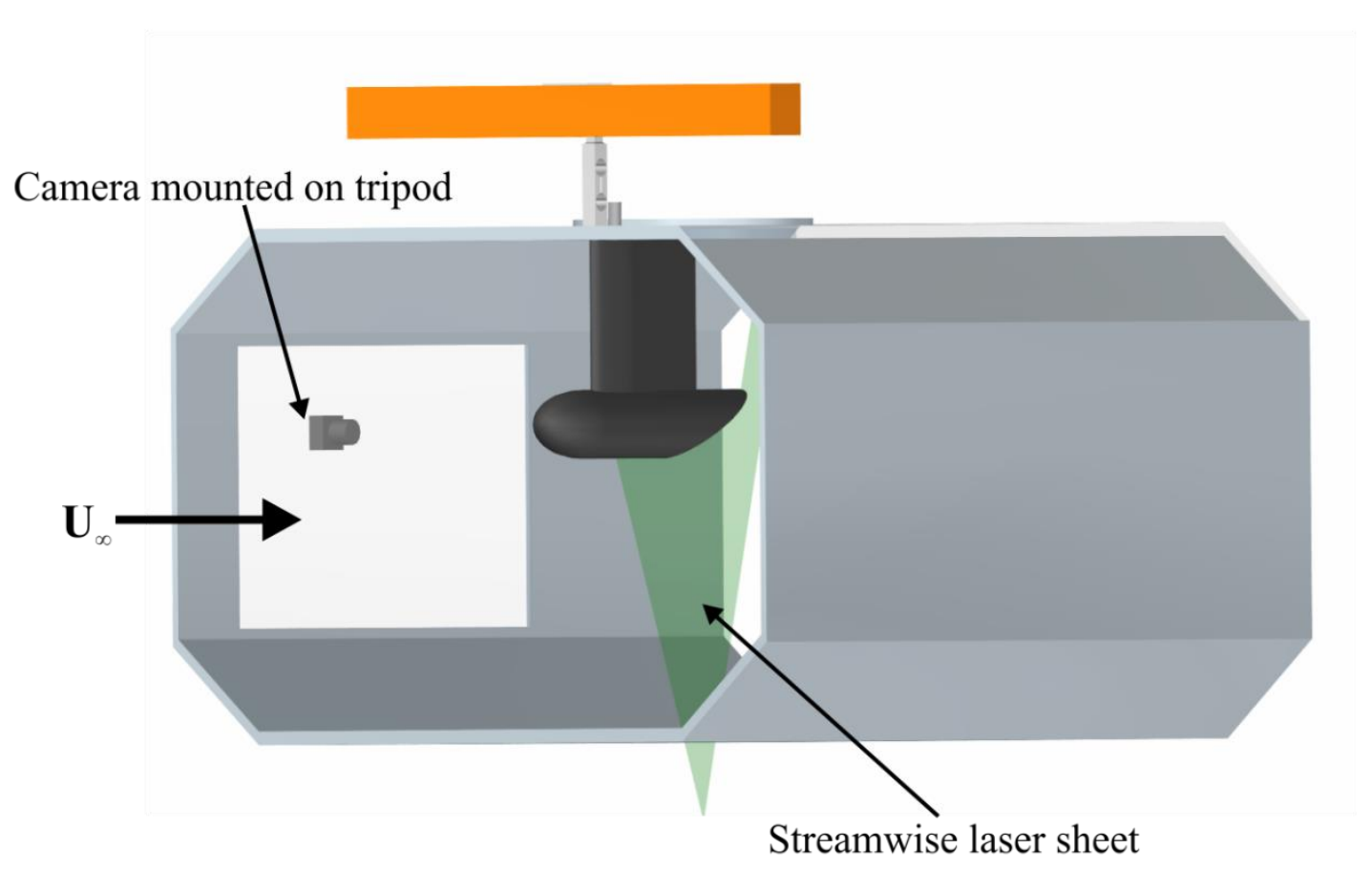

Figure 3. PIV Experimental setup. a) Crossflow plane, b) Streamwise $(z=0)$ plane. 


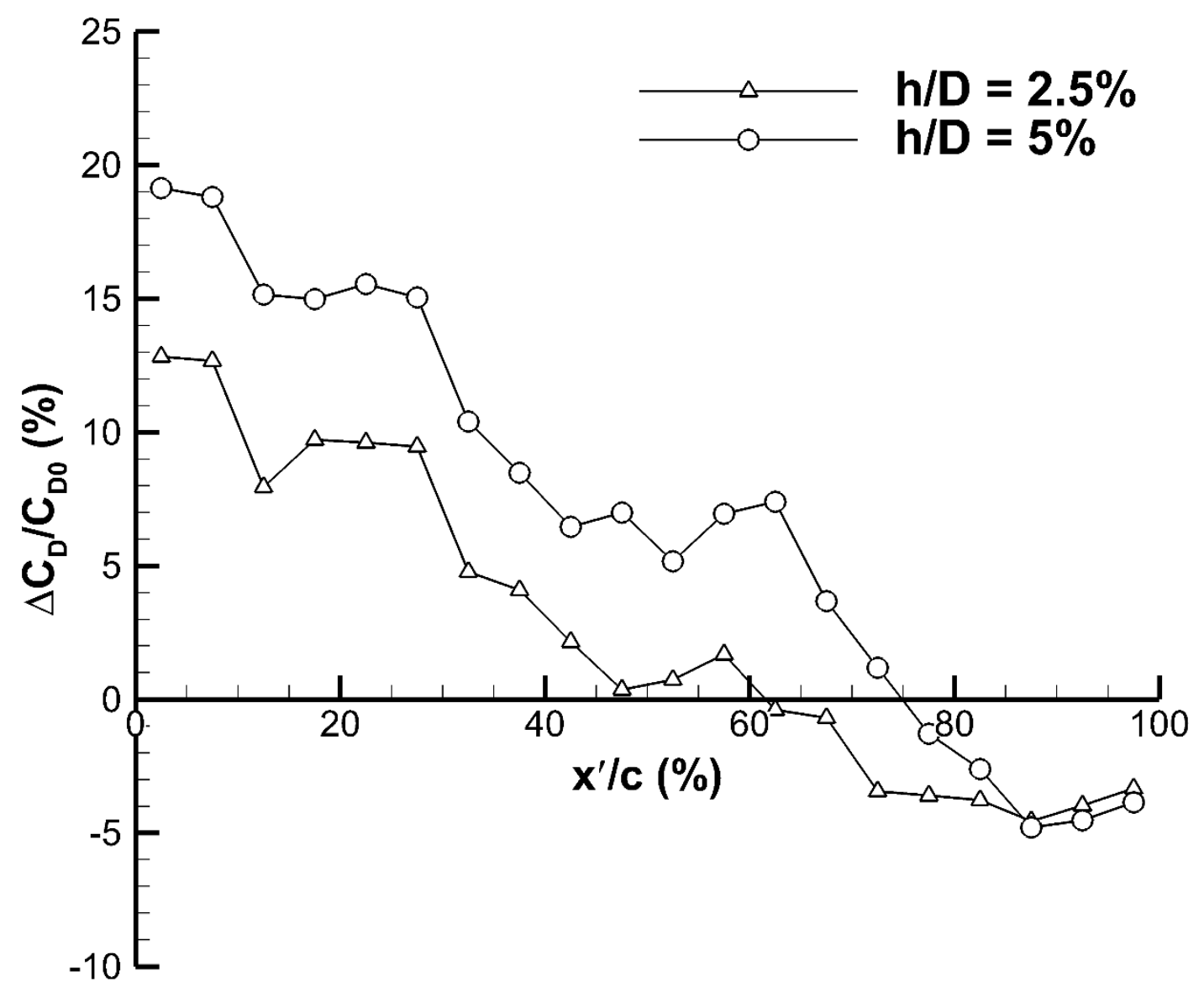

Figure 4. Variation of change in drag coefficient with spoiler location. 
a)


c)
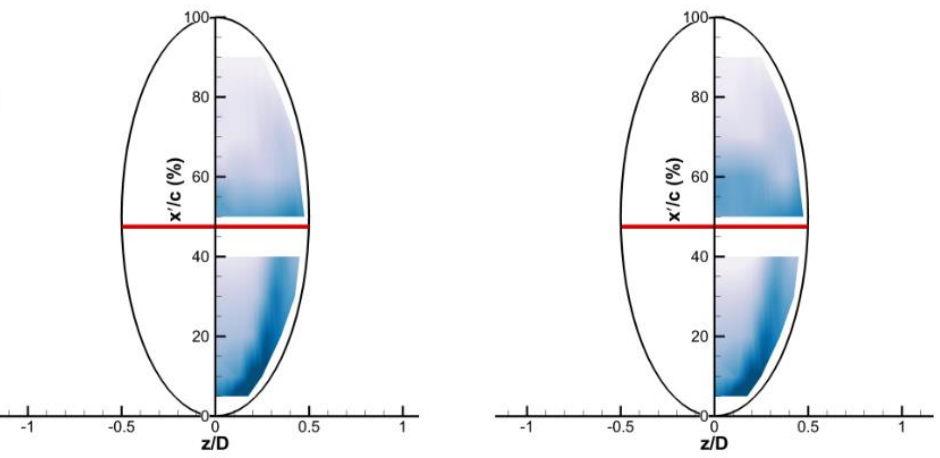

d)
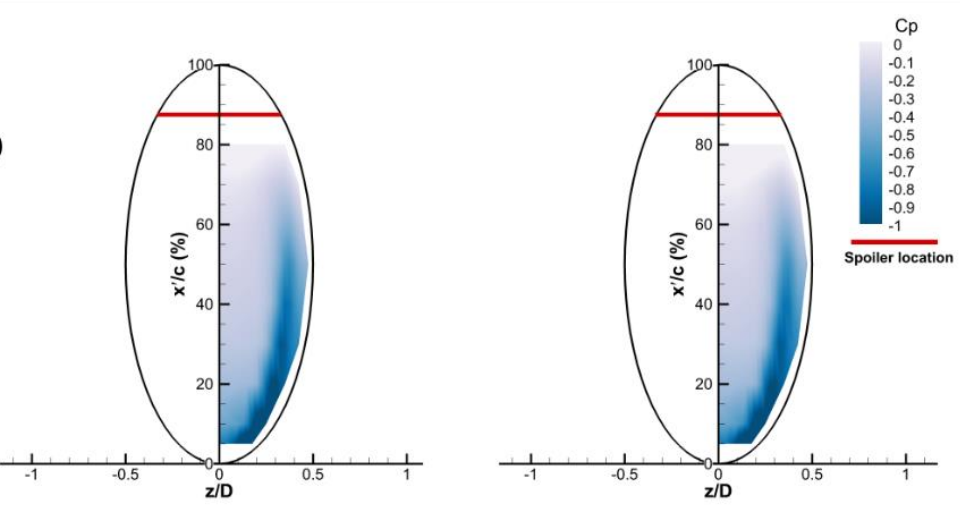

Figure 5. Surface pressure distributions for selected spoiler locations, $h / D=2.5 \%$ (left) and $h / D=5 \%$ (right). a) Baseline, b) $x_{s}^{\prime} / c=2.5 \%$, c) $x_{s}^{\prime} / c=47.5 \%$ and d) $x_{s}^{\prime} / c=87.5 \%$. Spoiler locations are denoted by horizontal red lines on each upsweep. 
a)

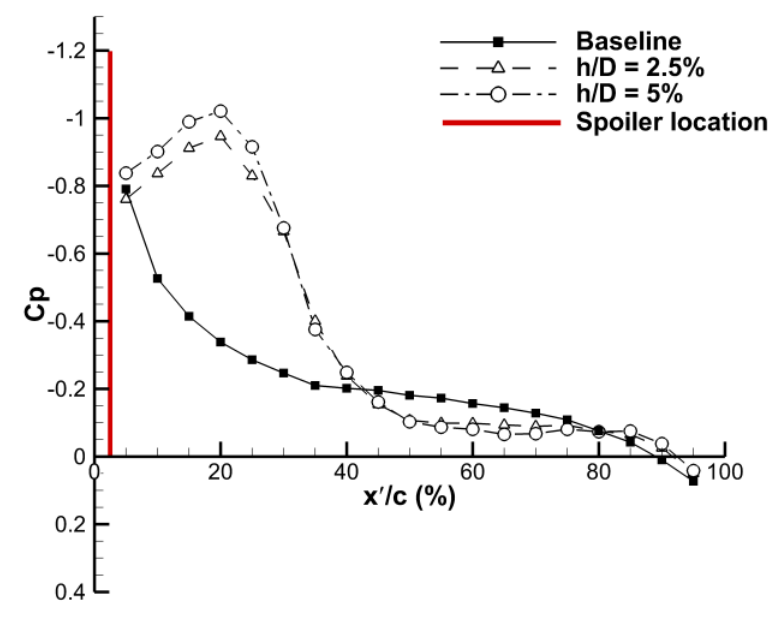

b)

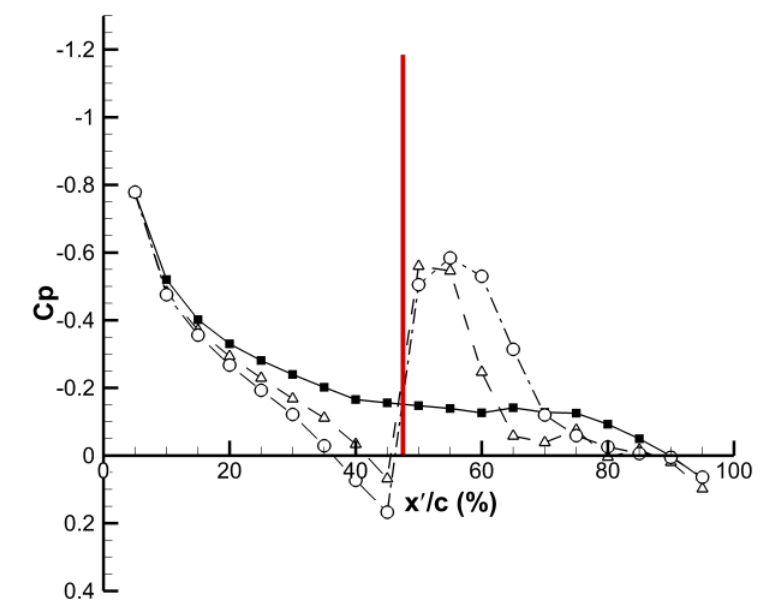

c)

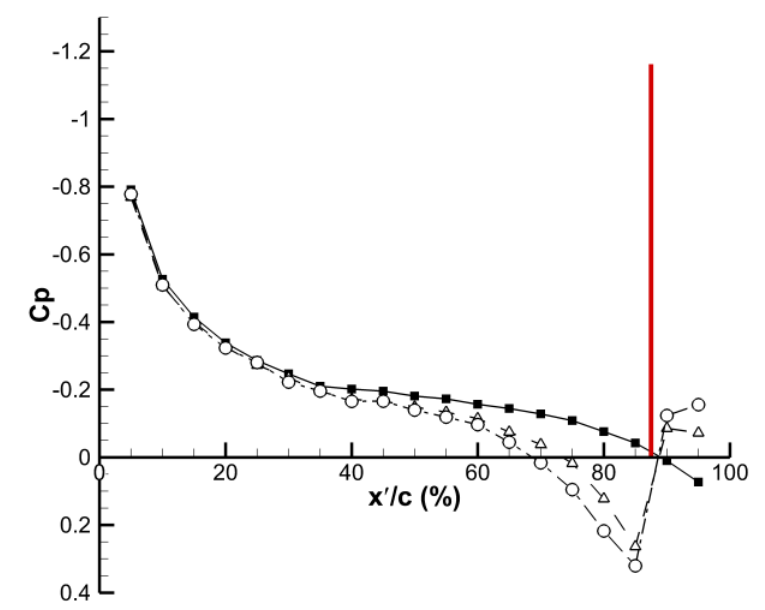

Figure 6. Centerline $(z=0)$ pressure. a) $x_{s}^{\prime} / c=2.5 \%$, b) $x_{s}^{\prime} / c=47.5 \%$ and c) $x_{s}^{\prime} / c=87.5 \%$. Spoiler locations are denoted by vertical red lines on each plot. 
a)

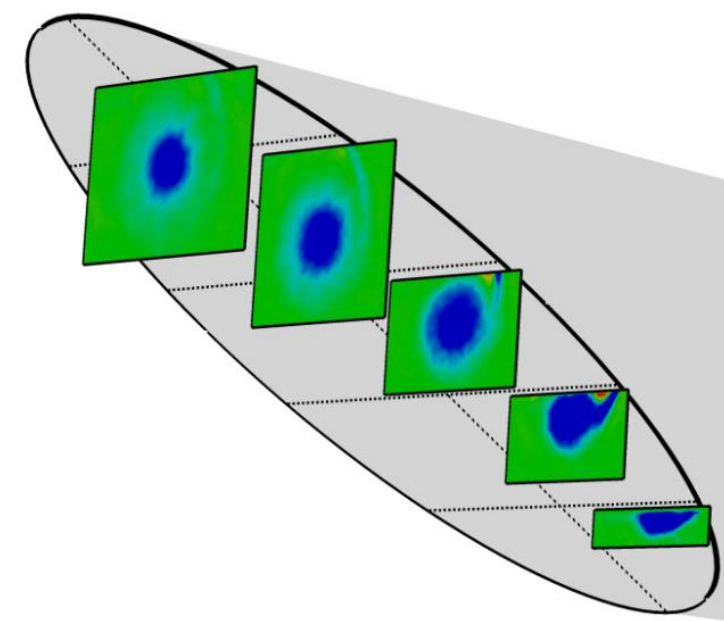

b)

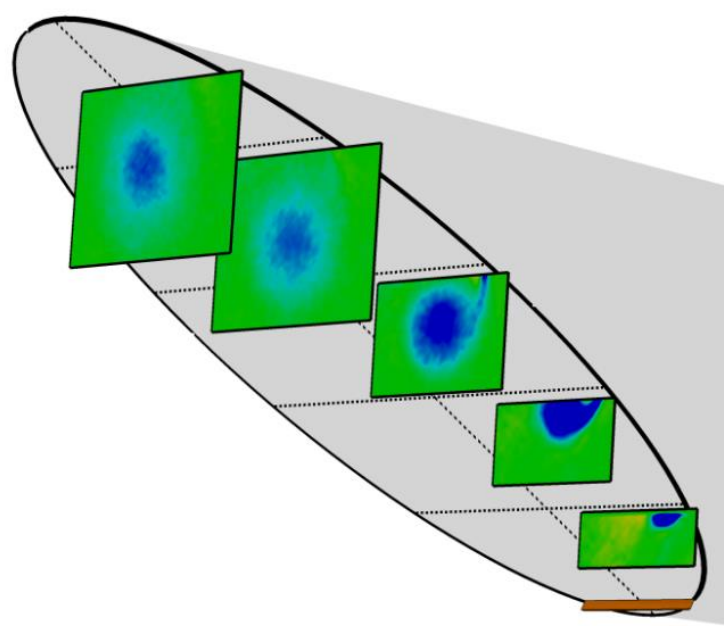

c)

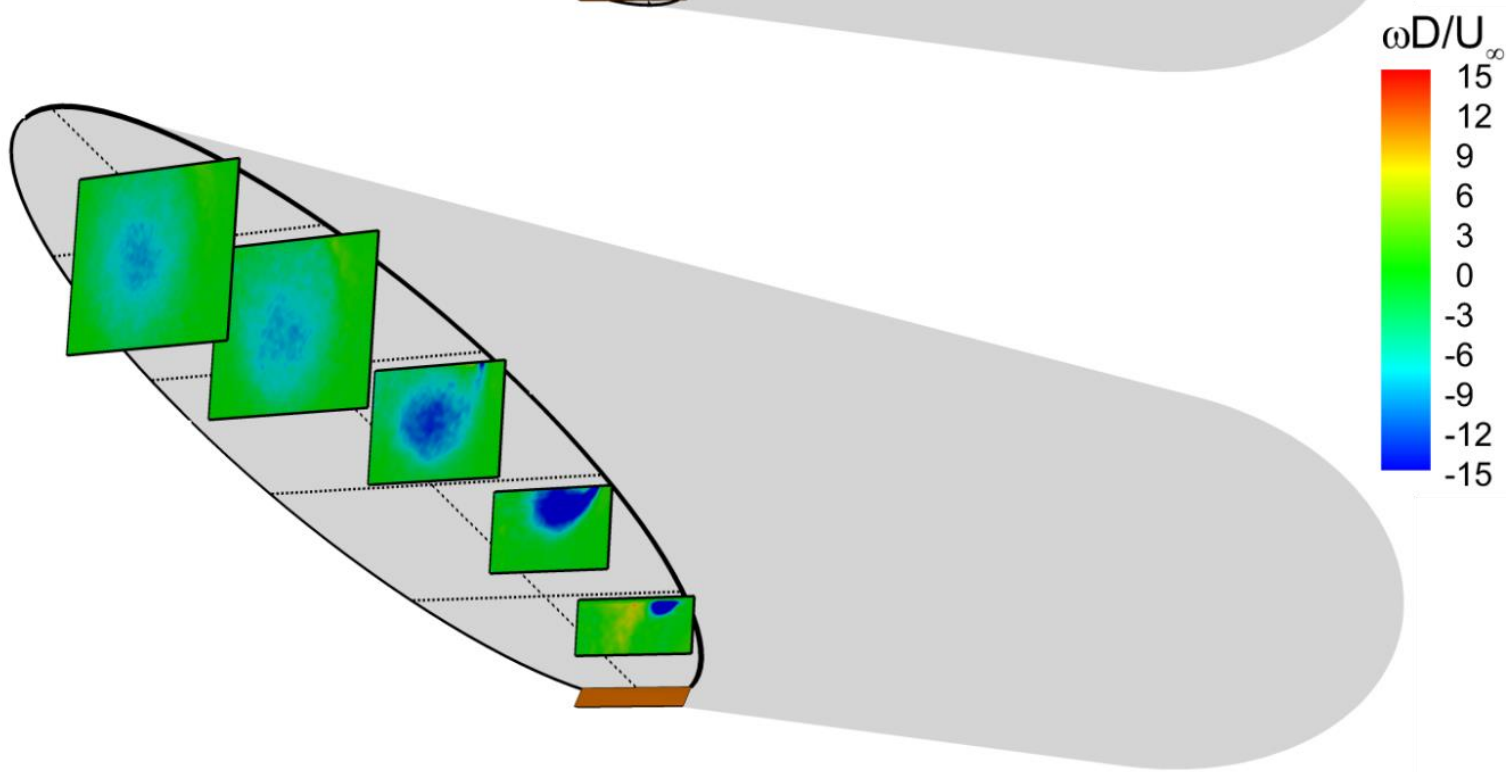

Figure 7. 3D perspective view of time-averaged vorticity for spoiler placed at $x_{s}^{\prime} / c=2.5 \%$, flow is from right to left. a) Baseline, b) $h / D=2.5 \%$ and c) $h / D=5 \%$. 
a)

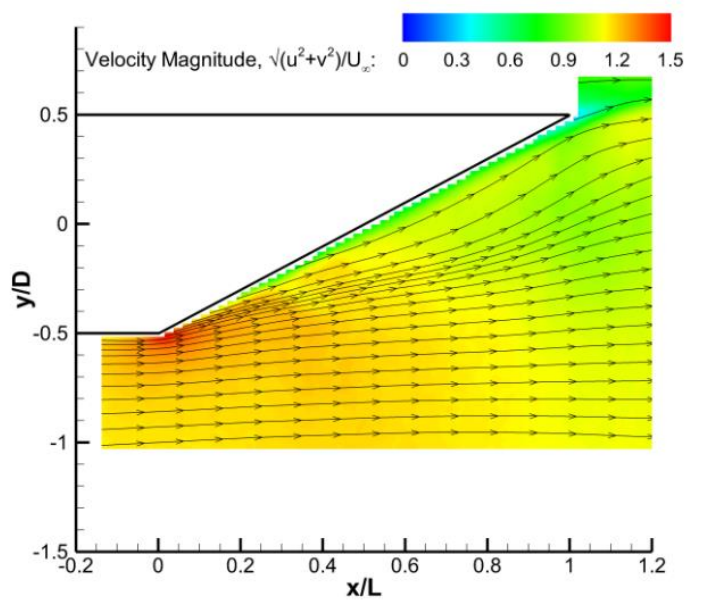

b)



c)

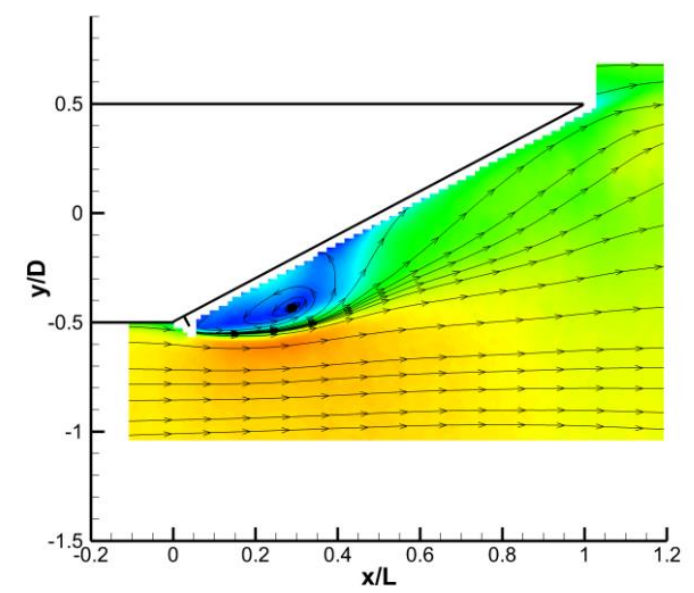

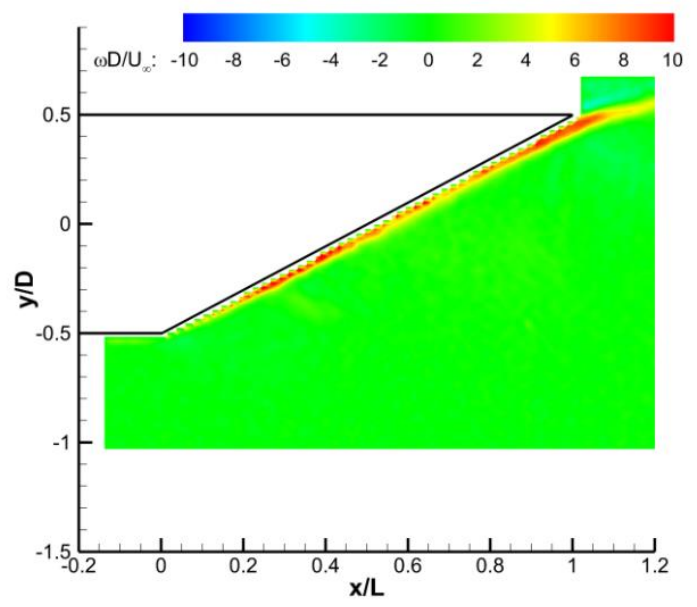
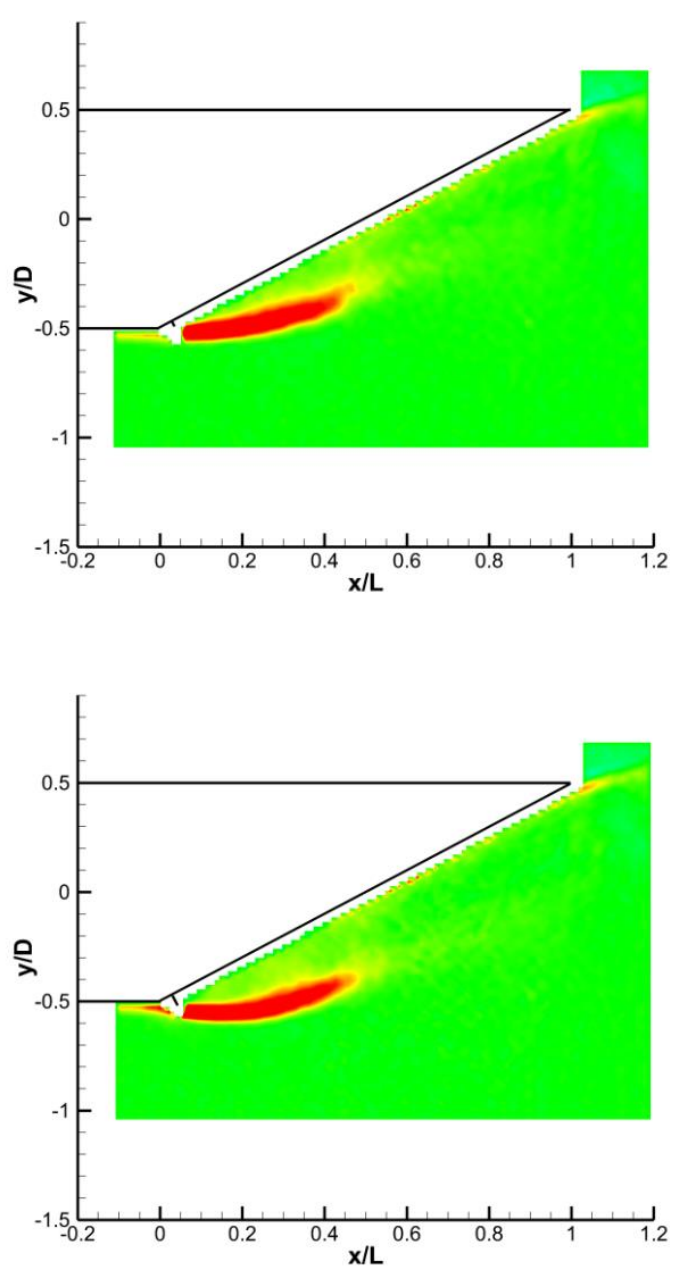

Figure 8. Time-averaged normalized velocity magnitude (left) and time-averaged vorticity (right) on the $z=0$ plane for spoiler placed at $x_{s}^{\prime} / c=2.5 \%$. Flow is from left to right. a) Baseline, b) $h / D=2.5 \%$ and

c) $h / D=5 \%$. 
a)



b)

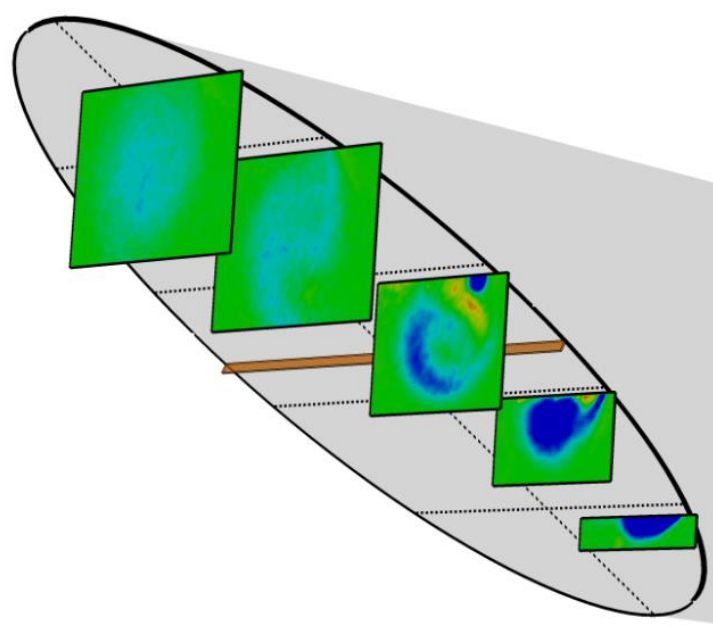

c)

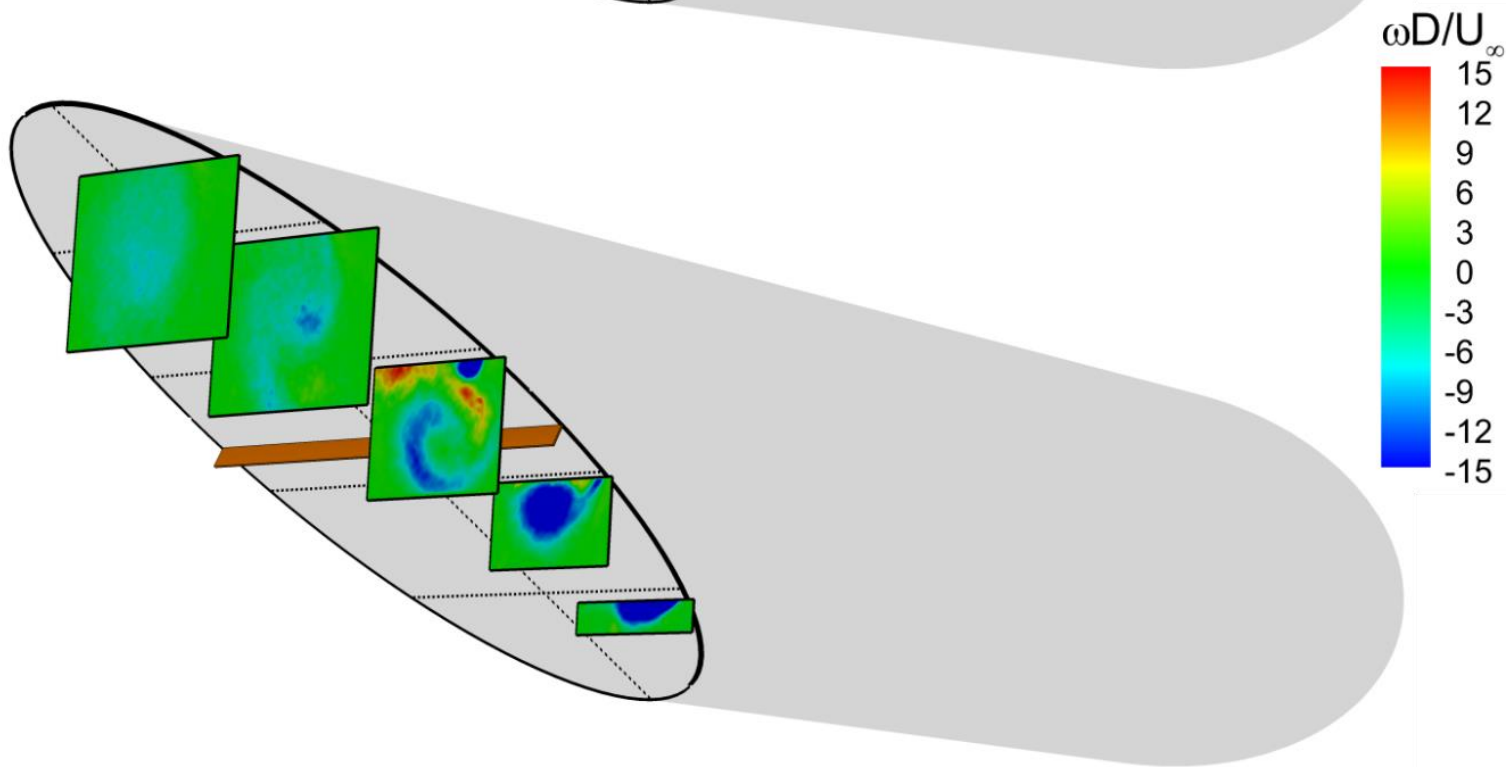

Figure 9. 3D perspective view of time-averaged vorticity for spoiler placed at $x_{s}^{\prime} / c=47.5 \%$, flow is from right to left. a) Baseline, b) $h / D=2.5 \%$ and c) $h / D=5 \%$. 
a)

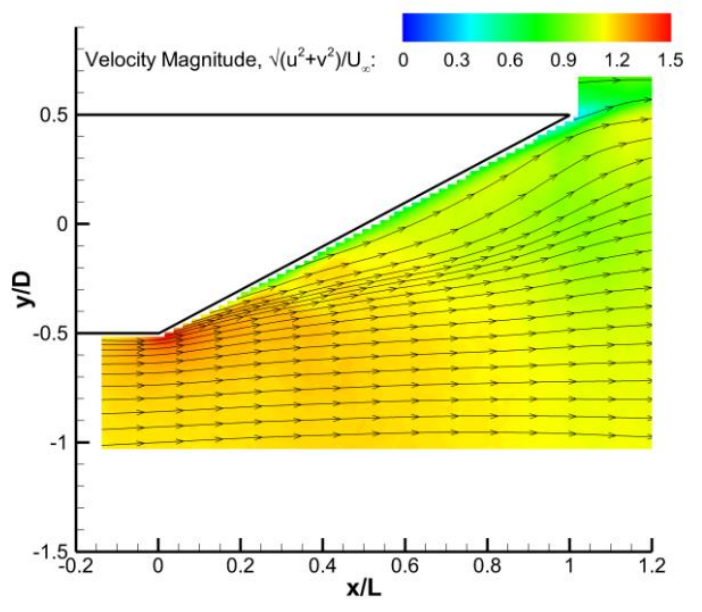

b)

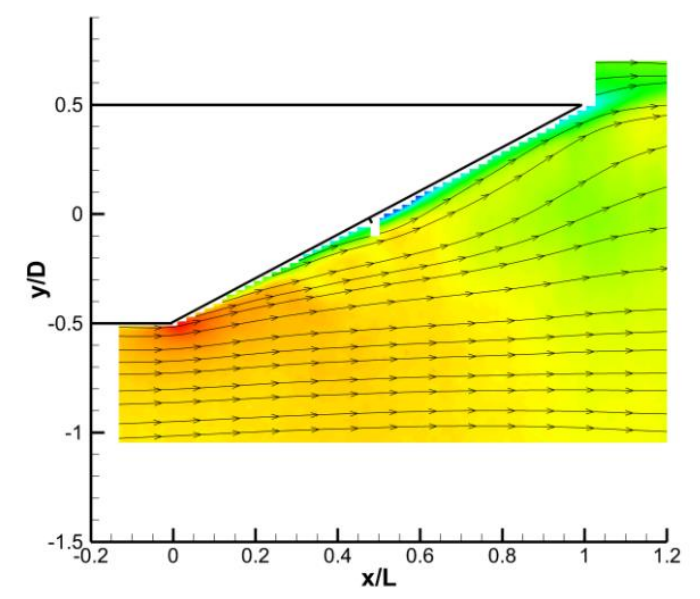

c)

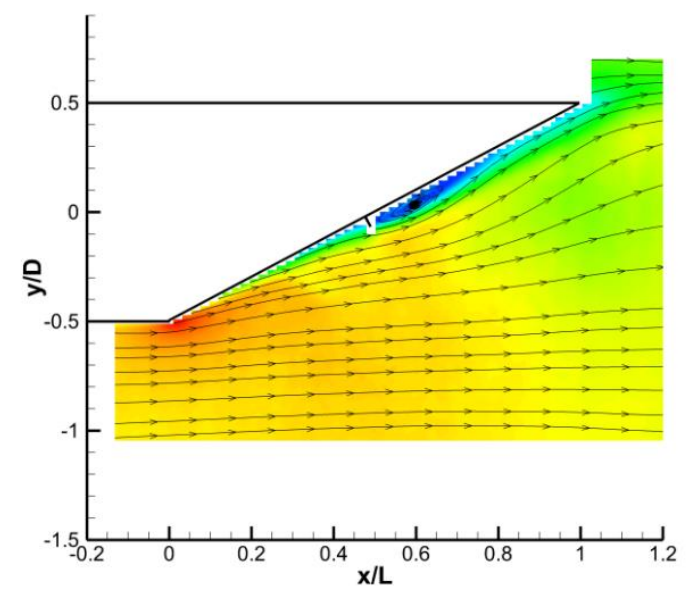

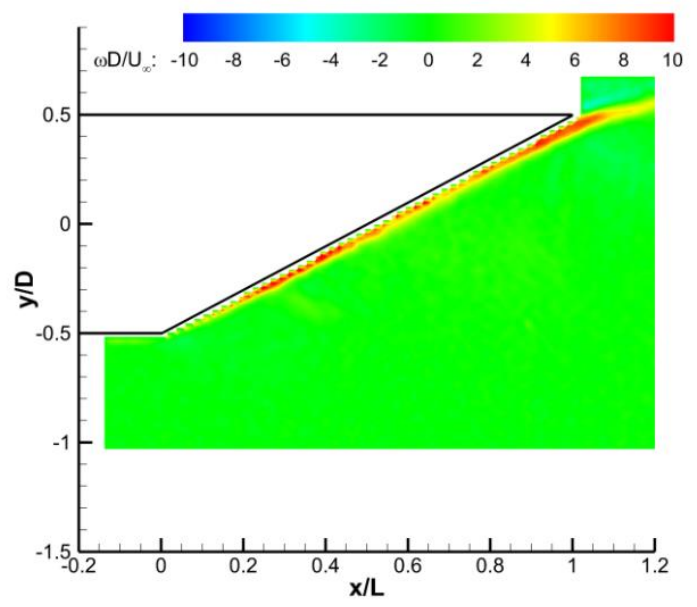
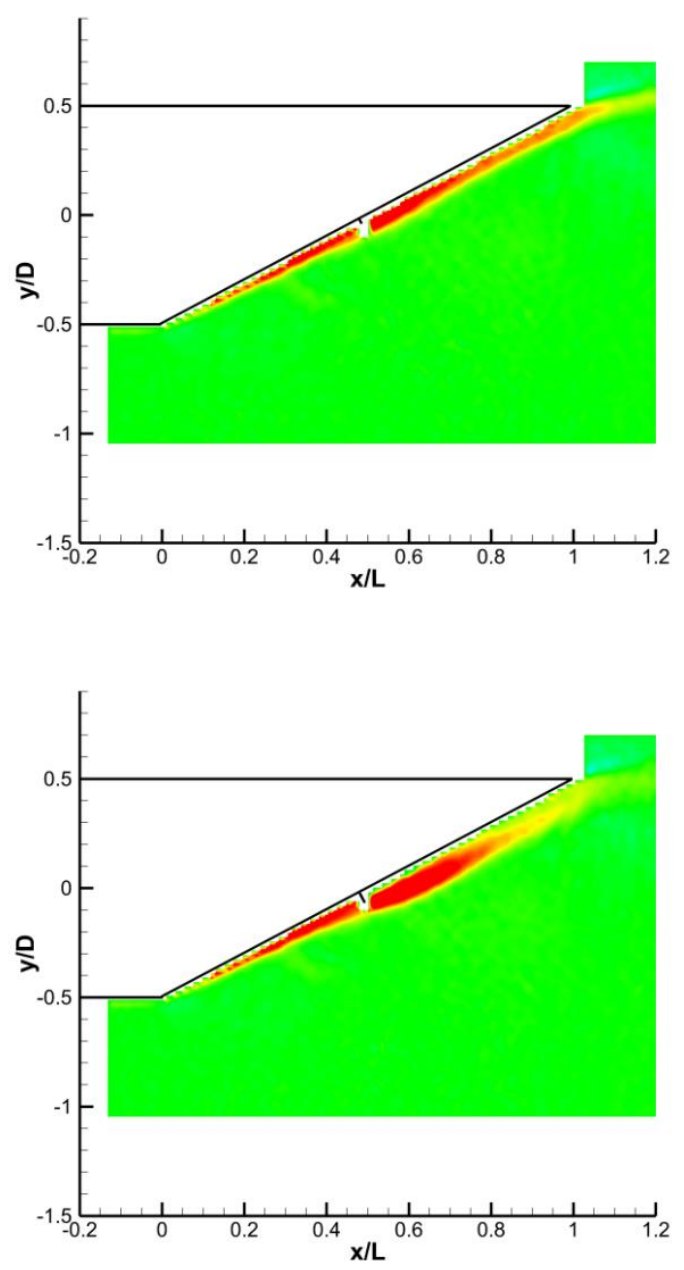

Figure 10. Time-averaged normalized velocity magnitude (left) and time-averaged vorticity (right) on the $z=0$ plane for spoiler placed at $x_{s}^{\prime} / c=47.5 \%$. Flow is from left to right. a) Baseline, b) $h / D=2.5 \%$ and c) $h / D=5 \%$. 
a)



b)

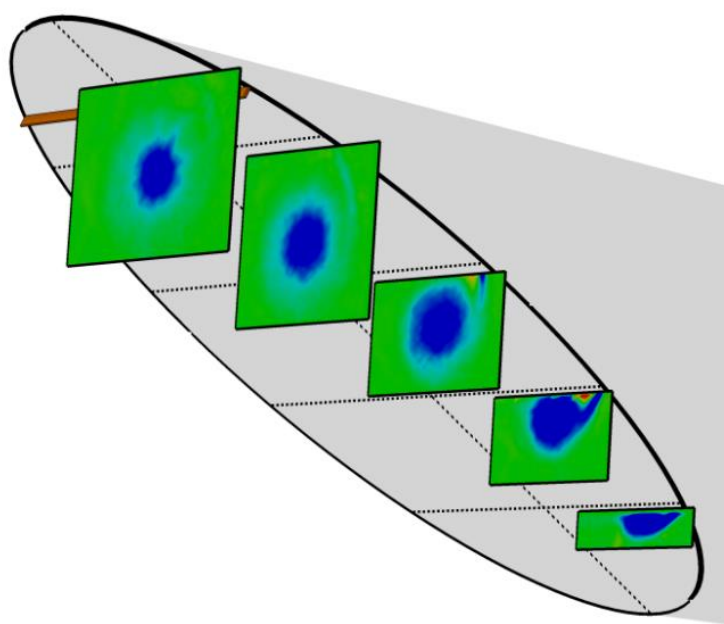

c)



Figure 11. 3D perspective view of time-averaged vorticity for spoiler placed at $x_{s}^{\prime} / c=87.5 \%$, flow is from right to left. a) Baseline, b) $h / D=2.5 \%$ and c) $h / D=5 \%$. 
a)

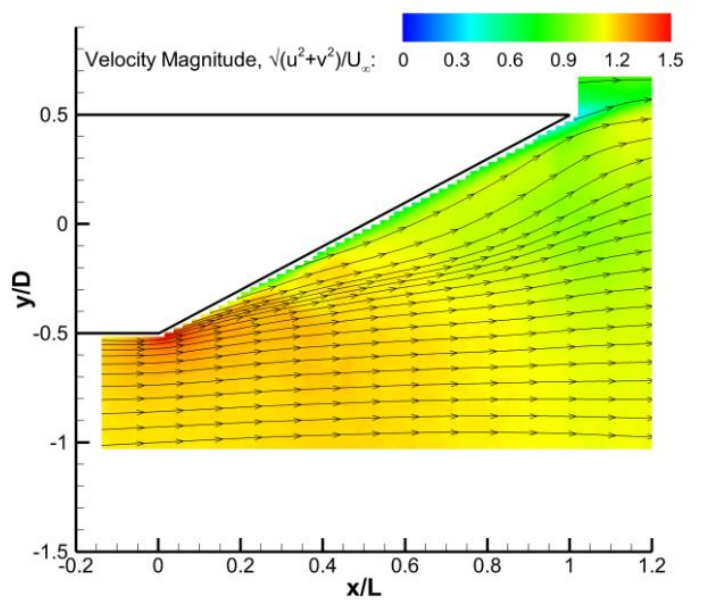

b)

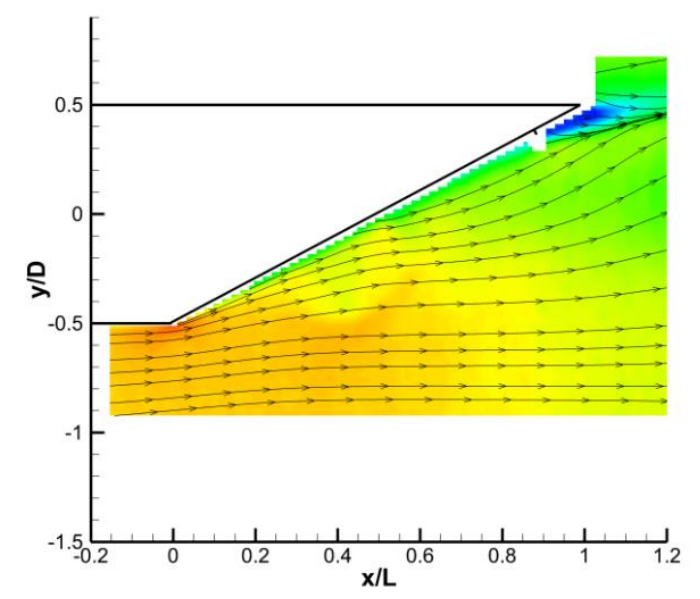

c)



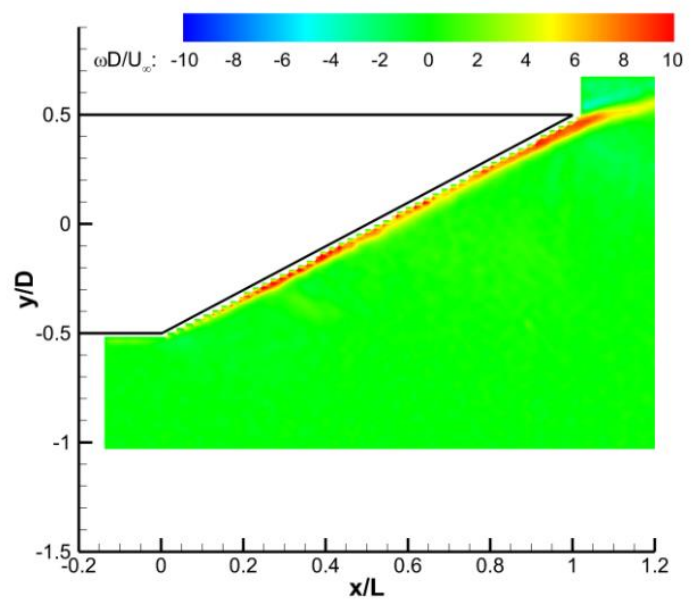
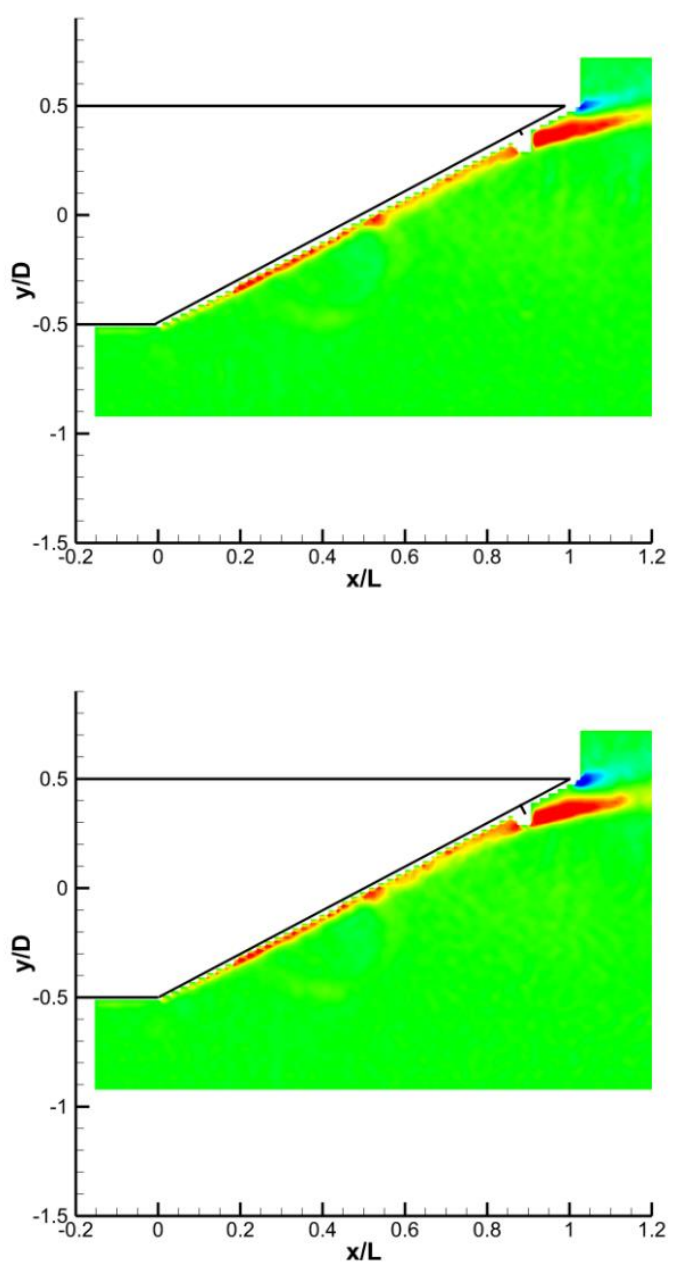

Figure 12. Time-averaged normalized velocity magnitude (left) and time-averaged vorticity (right) on the $z=0$ plane for spoiler placed at $x_{s}^{\prime} / c=87.5 \%$. Flow is from left to right. a) Baseline, b) $h / D=2.5 \%$ and

c) $h / D=5 \%$. 
a)



b)



c)

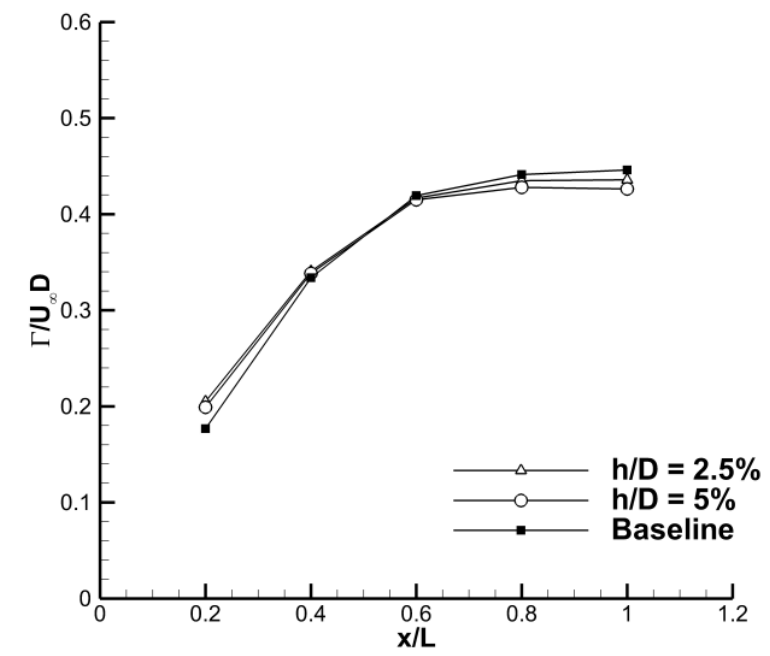

Figure 13. Variation of circulation. a) $x_{s}^{\prime} / c=2.5 \%$, b) $x_{s}^{\prime} / c=47.5 \%$ and c) $x_{s}^{\prime} / c=87.5 \%$ spoiler locations. 


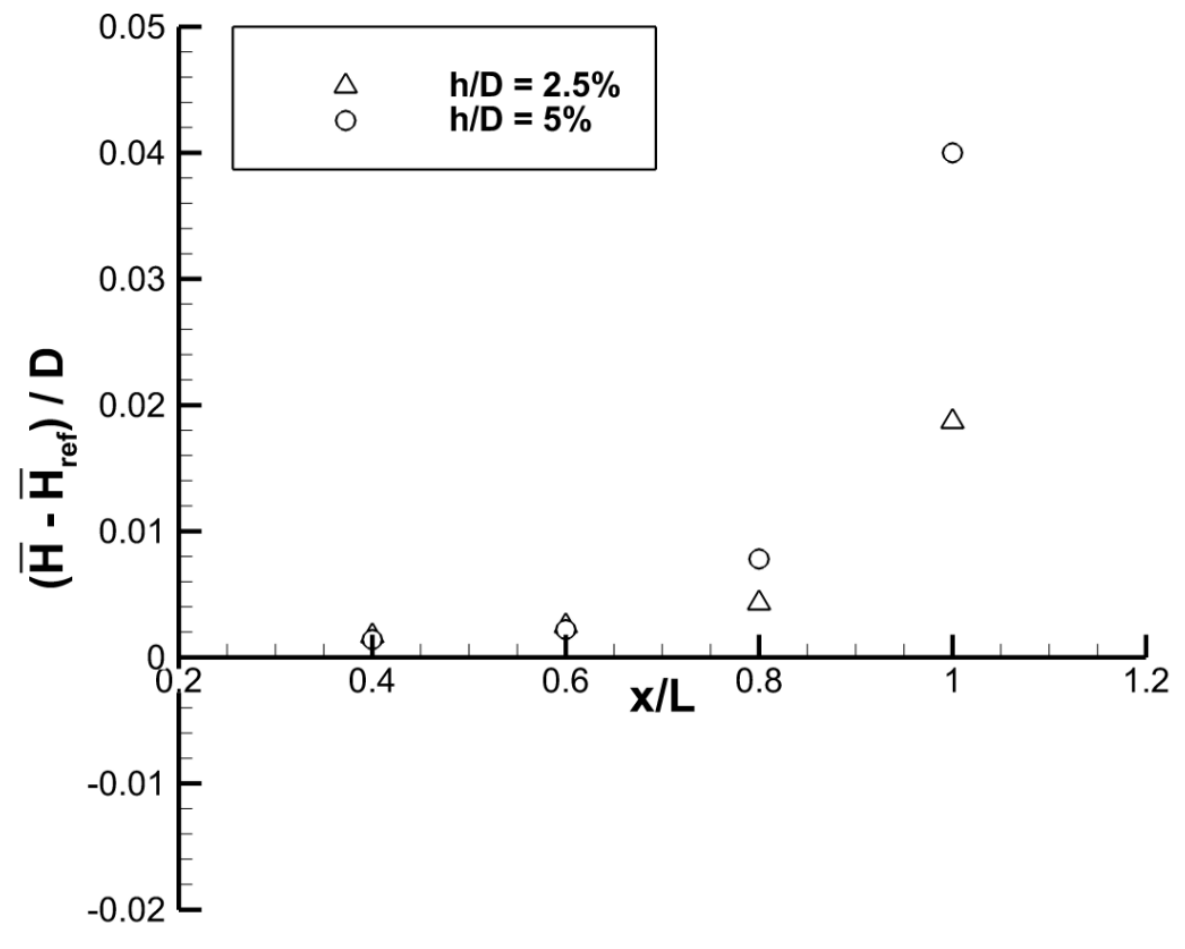

Figure 14. Vortex centroid locations for spoiler placed at $x_{s}^{\prime} / c=87.5 \%$. 
a)

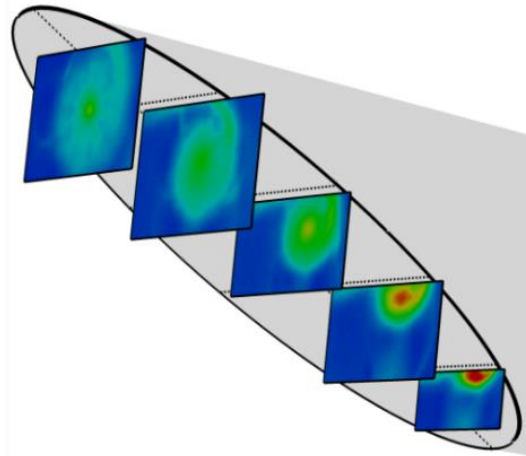

b)

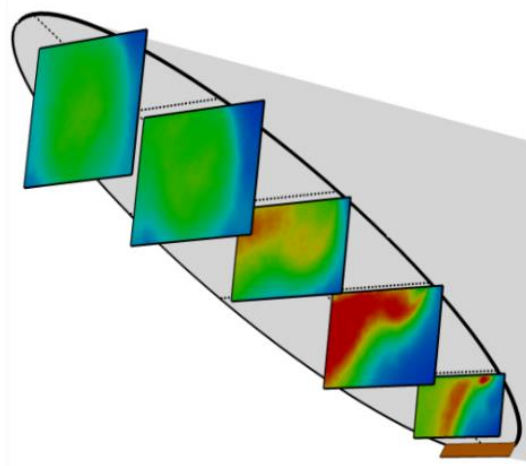

c)

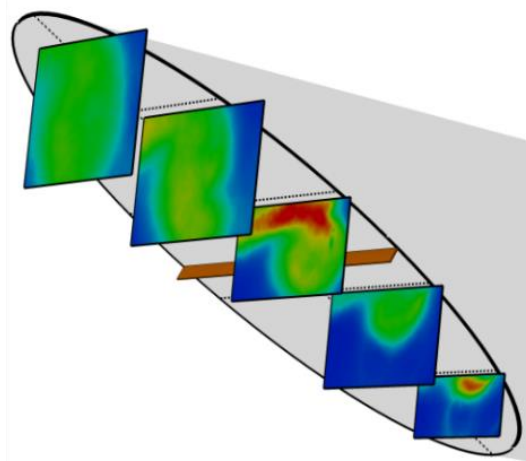

d)

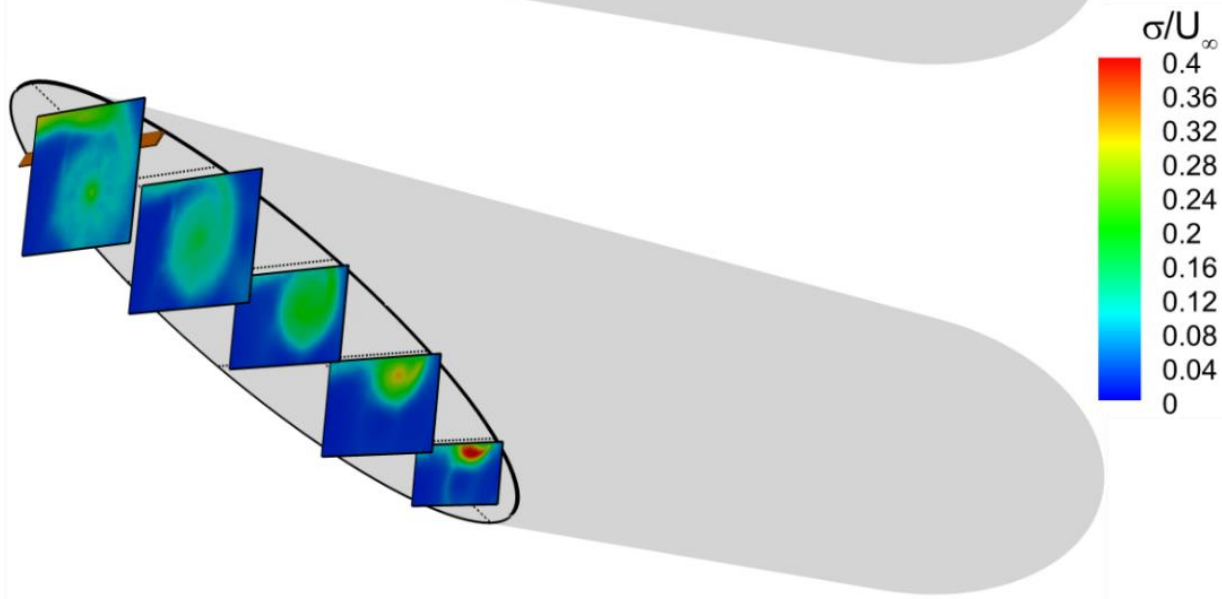

Figure 15. 3D perspective view of standard deviation of velocity, flow is from right to left. a) Baseline, b) $x_{s}^{\prime} / c=2.5 \%$, c) $x_{s}^{\prime} / c=47.5 \%$ and d) $x_{s}^{\prime} / c=87.5 \% . h / D=5 \%$ for all cases. 
a)



b)

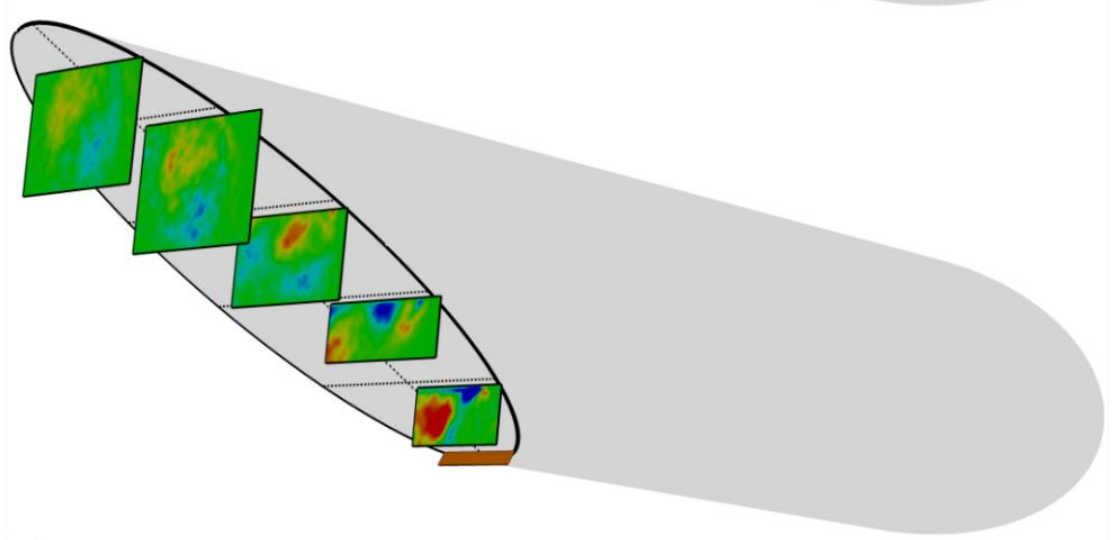

c)

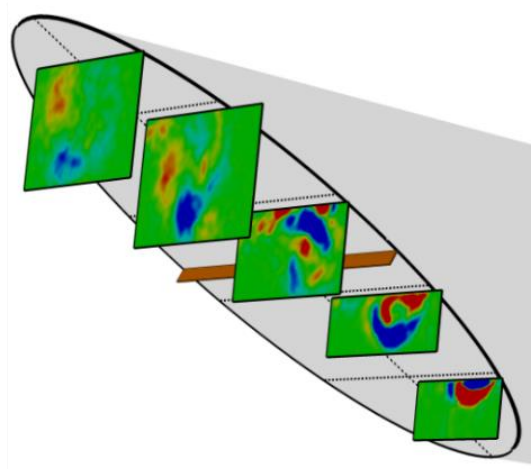

d)

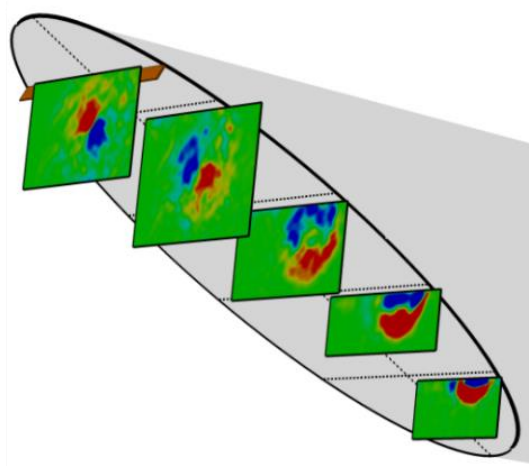

$\omega \mathrm{D} / \mathrm{U}^{\infty}$

0.08

0.064

0.048

0.032

0.016

0

$-0.016$

$-0.032$

$-0.048$

$-0.064$

$-0.08$

Figure 16. 3D perspective view of dominant POD mode, flow is from right to left. a) Baseline, b) $x_{s}^{\prime} / c=2.5 \%$, c) $x_{s}^{\prime} / c=47.5 \%$ and d) $x_{s}^{\prime} / c=87.5 \% . h / D=5 \%$ for all cases. 
a)

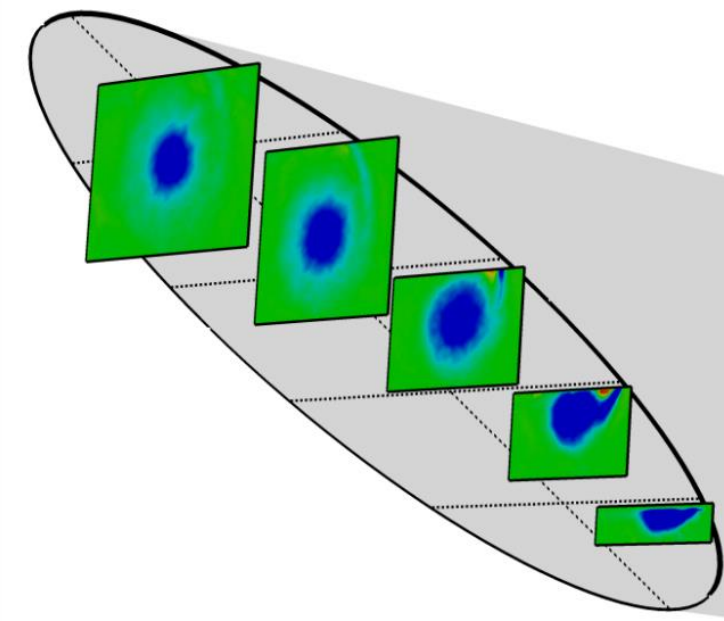

b)

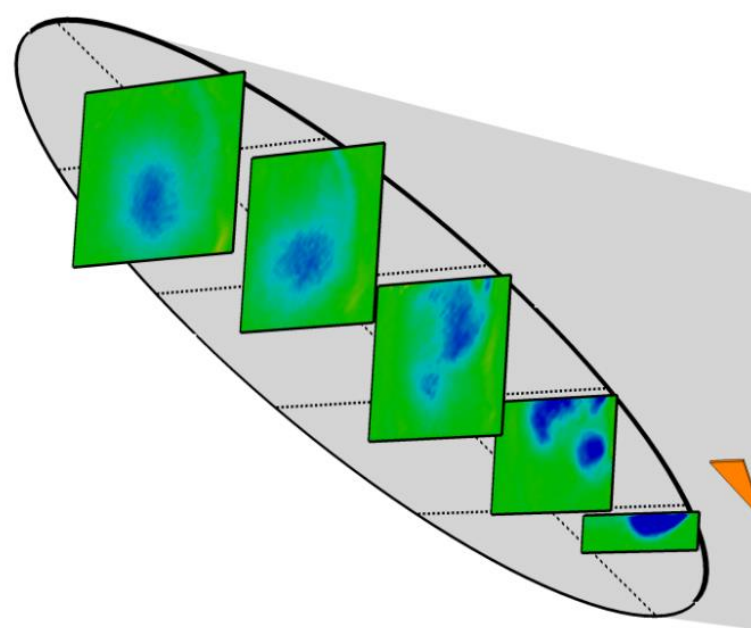

c)

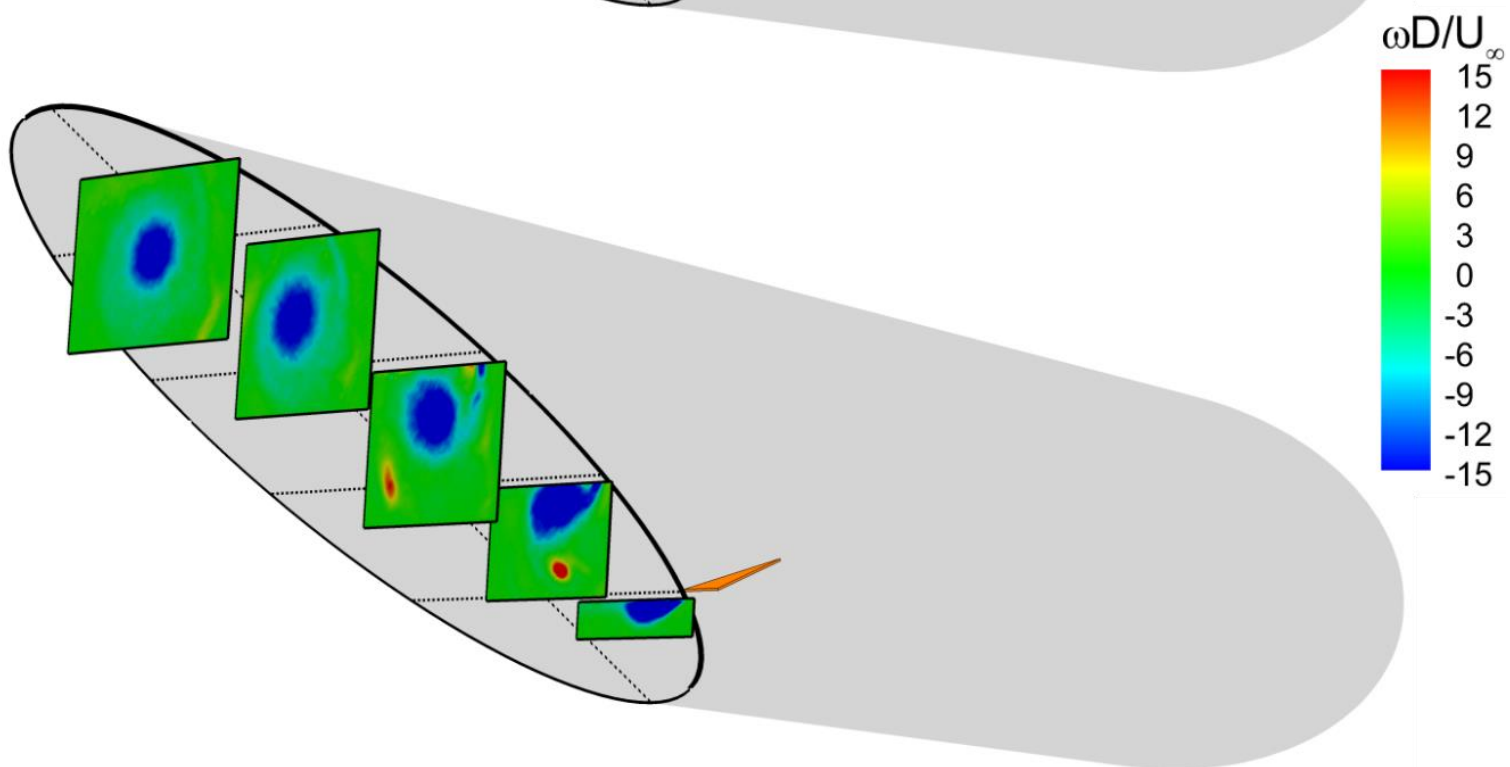

Figure 17. 3D perspective view of time-averaged vorticity for $\Lambda=\mathbf{7 0}^{\circ}$ sweep vortex generator with $\beta=20^{\circ}$, flow is from right to left. a) Baseline, b) Co-rotating and c) Counter-rotating cases. 


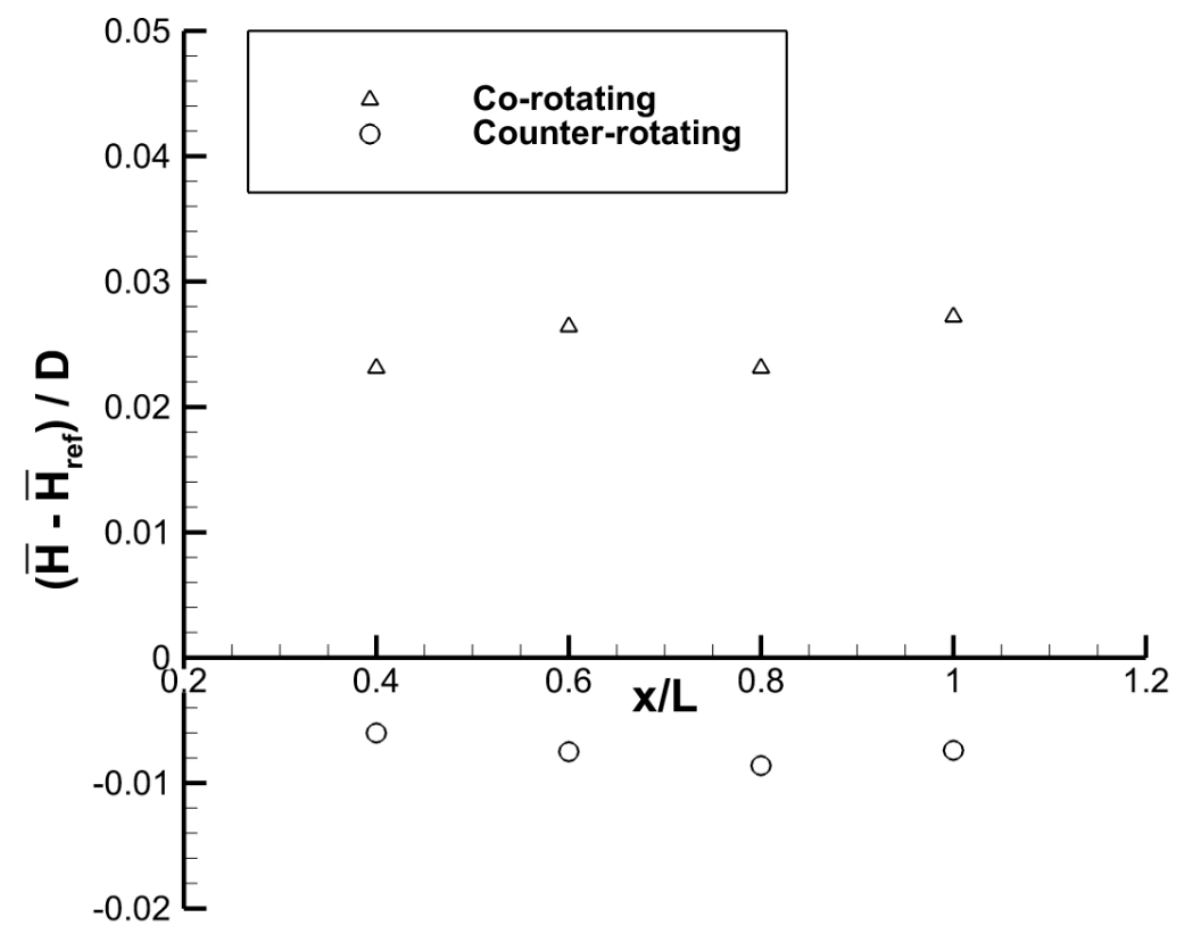

Figure 18. Vortex centroid locations for vortex generator configurations. 